\title{
Derivations and Deformations of $\delta$-Jordan Lie Supertriple Systems
}

\author{
Shengxiang Wang, ${ }^{1}$ Xiaohui Zhang, ${ }^{2}$ and Shuangjian Guo ${ }^{3}{ }^{3}$ \\ ${ }^{1}$ School of Mathematics and Finance, Chuzhou University, Chuzhou 239000, China \\ ${ }^{2}$ School of Mathematical Sciences, Qufu Normal University, Qufu 273165, China \\ ${ }^{3}$ School of Mathematics and Statistics, Guizhou University of Finance and Economics, Guiyang 550025, China
}

Correspondence should be addressed to Shuangjian Guo; shuangjianguo@126.com

Received 2 April 2019; Accepted 16 June 2019; Published 11 July 2019

Academic Editor: Francesco Toppan

Copyright (c) 2019 Shengxiang Wang et al. This is an open access article distributed under the Creative Commons Attribution License, which permits unrestricted use, distribution, and reproduction in any medium, provided the original work is properly cited.

Let $T$ be a $\delta$-Jordan Lie supertriple system. We first introduce the notions of generalized derivations and representations of $T$ and present some properties. Also, we study the low-dimensional cohomology and the coboundary operator of $T$, and then we investigate the deformations and Nijenhuis operators of $T$ by choosing some suitable cohomologies.

\section{Introduction}

Lie triple systems arose initially in Cartan's study of Riemannian geometry. Jacobson [1] first introduced Lie triple systems and Jordan triple systems in connection with problems from Jordan theory and quantum mechanics, viewing Lie triple systems as subspaces of Lie algebras that are closed relative to the ternary product. Lister [2] investigated notions of the radical, semisimplicity and solvability as defined for Lie triple systems and determined all simple Lie triple systems over an algebraically closed field. Later, the representation theory, the central extension, the deformation theory, bilinear forms, and the generalized derivation of Lie triple systems and Jordan triple systems have been developed; see [311].

In [12], Okubo and Kamiya introduced the notion of $\delta$ Jordan Lie triple system, where $\delta= \pm 1$, which is a generalization of both Lie triple systems $(\delta=1)$ and Jordan Lie triple systems $(\delta=-1)$. Later, Kamiya and Okubo [13] studied a construction of simple Jordan superalgebras from certain triple systems. Recently, Ma and Chen [14] discussed the cohomology theory, the deformations, Nijenhuis operators, abelian extensions, and $\mathrm{T}^{*}$-extensions of $\delta$-Jordan Lie triple system.
As a natural generalization of Lie triple systems, Okubo [15] introduced the notion of Lie supertriple systems in the study of Yang-Baxter equations. Lie supertriple systems have many applications in high energy physics, and many important results on Lie supertriple systems have been obtained; see $[13,15-17]$. In [12], Okubo and Kamiya introduced the notion of $\delta$-Jordan Lie supertriple system (they still call it Jordan Lie triple system); they presented some nontrivial examples and discussed their quasiclassical property. In the present paper, we hope to study generalized derivations, cohomology theories, and deformations of $\delta$-Jordan Lie supertriple systems.

This paper is organized as follows. In Section 2, we recall the definition of $\delta$-Jordan Lie supertriple systems and construct a kind of $\delta$-Jordan Lie supertriple systems. Also, we study generalized derivation algebra of a $\delta$-Jordan Lie supertriple system. In Section 3, we introduce notions of the representation and low-dimensional cohomology of a $\delta$ Jordan Lie supertriple system. In Section 4, we consider the theory of deformations of a $\delta$-Jordan Lie supertriple system by choosing a suitable cohomology. In Section 5, we study Nijenhuis operators for a $\delta$-Jordan Lie supertriple system to describe trivial deformations. 


\section{Generalized Derivations of $\delta$-Jordan Lie Supertriple Systems}

In this section, we start by recalling the definition of $\delta$-Jordan Lie supertriple systems and then we study its generalized derivations.

Definition 1 ([12]). A $\delta$-Jordan Lie supertriple system is a $Z_{2}$ graded vector space $T$ together with a triple linear product $[\cdot, \cdot, \cdot]: V \otimes V \otimes V \longrightarrow V$ satisfying

$$
\begin{aligned}
& \text { (1) }|[a, b, c]|=(|a|+|b|+|c|)(\bmod 2) ; \\
& \text { (2) }[b, a, c]=-\delta(-1)^{|a||b|}[a, b, c] ; \\
& \text { (3) }(-1)^{|a||c|}[a, b, c]+(-1)^{|b||a|}[b, c, a] \\
& +(-1)^{|c||b|}[c, a, b]=0 ; \\
& \text { (4) }[a, b,[c, d, e]]=[[a, b, c], d, e] \\
& +(-1)^{|c|(|a|+|b|)}[c,[a, b, d], e] \\
& +\delta(-1)^{(|a|+|b|)(|c|+|d|)}[c, d,[a, b, e]],
\end{aligned}
$$

for all $a, b, c, d, e \in T$, where $\delta= \pm 1$ and $|a|$ denotes the degree of the element $a \in T$.

Remark 2. Clearly, $T_{\overline{0}}$ is an ordinary $\delta$-Jordan Lie triple system in [14]. The case of $\delta=1$ defines, especially, a Lie supertriple system while the other case of $\delta=-1$ may be termed an anti-Lie supertriple system as in [18].

Example $3([12])$. Let $(T,[\cdot, \cdot])$ be a $\delta$-Jordan Lie superalgebra. Then $(T,[\cdot, \cdot, \cdot])$ becomes a $\delta$-Jordan Lie supertriple system, where $[a, b, c]=[[a, b], c]$, for all $a, b, c \in T$.

Example 4. Let $T$ be a $\delta$-Jordan Lie supertriple system and $t$ an indeterminate. Set $T^{\prime}=\left\{\sum_{i \geq 0} x \otimes t^{i} \mid x \in T\right\}$; then $T^{\prime}$ is a $\delta$-Jordan Lie supertriple system with a triple linear product $[\cdot, \cdot, \cdot]^{\prime}$ defined by

$$
\left[a \otimes t^{i}, b \otimes t^{j}, c \otimes t^{k}\right]^{\prime}=[a, b, c] \otimes t^{i+j+k},
$$

for all $a \otimes t^{i}, b \otimes t^{j}, c \otimes t^{k} \in T^{\prime}$, where $\left|a \otimes t^{i}\right|=|a|$.

Definition 5. Let $T$ be a $\delta$-Jordn Lie supertriple system and $k$ a nonnegative integer. A homogeneous linear map $D: T \longrightarrow T$ is said to be a homogeneous $k$-derivation of $T$ if it satisfies

$$
\begin{aligned}
\delta^{k}[D & (a), b, c]+\delta^{k}(-1)^{|D||a|}[a, D(b), c] \\
& +\delta^{k}(-1)^{|D|(|a|+|b|)}[a, b, D(c)]=D([a, b, c]),
\end{aligned}
$$

for all $a, b, c \in T$, where $|D|$ denotes the degree of $D$.

We denote by $\operatorname{Der}(T)=\bigoplus_{k \geq 0} \operatorname{Der}_{k}(T)$, where $\operatorname{Der}_{k}(T)$ is the set of all homogeneous $k$-derivations of $T$. Obviously, $\operatorname{Der}(T)$ is a subalgebra of $\operatorname{End}(T)$ and has a normal Lie superalgebra structure via the bracket product

$$
\left[D, D^{\prime}\right]=D D^{\prime}-(-1)^{\left|D \| D^{\prime}\right|} D^{\prime} D .
$$

Definition 6. Let $T$ be a $\delta$-Jordan Lie supertriple system and $k$ a nonnegative integer. $D \in \operatorname{End}_{\bar{s}}(T)$ is said to be a homogeneous generalized $k$-derivation of $T$, if there exist three endomorphisms $D^{\prime}, D^{\prime \prime}, D^{\prime \prime \prime} \in \operatorname{End}_{\bar{s}}(T)$ such that

$$
\begin{aligned}
& \delta^{k}[D(a), b, c]+\delta^{k}(-1)^{s|a|}\left[a, D^{\prime}(b), c\right] \\
& \quad+\delta^{k}(-1)^{s(|a|+|b|)}\left[a, b, D^{\prime \prime}(c)\right]=D^{\prime \prime \prime}([a, b, c]),
\end{aligned}
$$

for all $a, b, c \in T$.

Definition 7. Let $T$ be a $\delta$-Jordan Lie supertriple system and $k$ a nonnegative integer. $D \in \operatorname{End}_{\bar{s}}(T)$ is said to be a homogeneous $k$-quasiderivation of $T$, if there exists an endomorphism $D^{\prime} \in \operatorname{End}_{\bar{s}}(T)$ such that

$$
\begin{aligned}
& \delta^{k}[D(a), b, c]+\delta^{k}(-1)^{s|a|}[a, D(b), c] \\
& \quad+\delta^{k}(-1)^{s(|a|+|b|)}[a, b, D(c)]=D^{\prime}([a, b, c]),
\end{aligned}
$$

for all $a, b, c \in T$.

Let $G \operatorname{Der}(T)$ and $Q \operatorname{Der}(T)$ be the sets of homogeneous generalized $k$-derivations and of homogeneous $k$ quasiderivations, respectively. That is,

$$
\begin{aligned}
& \operatorname{GDer}(T)=\bigoplus_{k \geq 0} \operatorname{GDer}_{k}(T), \\
& \operatorname{QDer}(T)=\bigoplus_{k \geq 0} \operatorname{QDer}_{k}(T) .
\end{aligned}
$$

Definition 8. Let $T$ be a $\delta$-Jordan Lie supertriple system and $k$ a nonnegative integer. The $k$-centroid of $T$ is the space of linear transformations on $T$ given by

$$
\begin{aligned}
C^{k} & (T)=\left\{D \in \operatorname{End}(T) \mid \delta^{k}[D(a), b, c]\right. \\
& =\delta^{k}(-1)^{|D||a|}[a, D(b), c] \\
& \left.=\delta^{k}(-1)^{|D|(|a|+|b|)}[a, b, D(c)]=D([a, b, c])\right\} .
\end{aligned}
$$

We denote $C(T)=\bigoplus_{k \geq 0} C^{k}(T)$ and call it the centroid of $T$.

Definition 9. Let $T$ be a $\delta$-Jordan Lie supertriple system. The quasicentroid of $T$ is the space of linear transformations on $T$ given by

$$
\begin{gathered}
\text { QC }(T)=\{D \in \operatorname{End}(T) \mid D([a, b, c]) \\
=[D(a), b, c], \forall a, b, c \in T\} .
\end{gathered}
$$

Remark 10. Let $T$ be a $\delta$-Jordan Lie supertriple system. Then $Q C(T) \subseteq C(T)$.

For any $D \in Q C(T)$ and $a, b, c \in T$, we have

$$
\begin{aligned}
D([a, b, c]) & =[D(a), b, c]=(-1)^{|D \|| a \mid}[a, D(b), c] \\
& =(-1)^{|D|(|a|+|b|)}[a, b, D(c)] .
\end{aligned}
$$


In fact, by the definition of the $\delta$-Jordan Lie supertriple system, we have

$$
\begin{aligned}
D([a, b, c]) & =-\delta(-1)^{|a||b|} D([b, a, c]) \\
& =-\delta(-1)^{|a||b|}[D(b), a, c] \\
& =\delta^{2}(-1)^{|a||b|}(-1)^{|a| \mid(b|+| D \mid)}[a, D(b), c] \\
& =(-1)^{|D \|||a|}[a, D(b), c] .
\end{aligned}
$$

Similarly, we have

$$
\begin{aligned}
&(-1)^{|a|(|D|+|c|)}[a, b, D(c)] \\
&=-(-1)^{|a||b|}[b, D(c), a] \\
&-(-1)^{|b|(|D|+|c|)}[D(c), a, b] \\
&=-(-1)^{|b||| a|+| D \mid)} D([b, c, a]) \\
&-(-1)^{|b|(|D|+|c|)} D([c, a, b]) \\
&=-(-1)^{|b||D|} D\left((-1)^{|b||a|}[b, c, a]+(-1)^{|c||b|}[c, a, b]\right) \\
&=(-1)^{|b||D|} D\left((-1)^{|a||c|}[a, b, c]\right) .
\end{aligned}
$$

It follows that $[a, b, D(c)]=(-1)^{|D|(|a|+|b|)}[a, b, D(c)]$.

Definition 11. Let $T$ be a $\delta$-Jordan Lie supertriple system. $D \in$ $\operatorname{End}(T)$ is said to be a central derivation of $T$ if

$$
D([a, b, c])=[D(a), b, c]=0,
$$

for all $a, b, c \in T$. Denote the set of all central derivations by $Z \operatorname{Der}(T)$.

Remark 12. Let $T$ be a $\delta$-Jordan Lie supertriple system. Then

$$
\begin{aligned}
Z \operatorname{Der}(T) & \subseteq \operatorname{Der}(T) \subseteq Q \operatorname{Der}(T) \subseteq G \operatorname{Der}(T) \\
& \subseteq \operatorname{End}(T) .
\end{aligned}
$$

Definition 13. Let $T$ be a $\delta$-Jordan Lie supertriple system. If $Z(T)=\{a \in T \mid[a, b, c]=0, \forall b, c \in T\}$, then $Z(T)$ is called the center of $T$.

Proposition 14. Let $T$ be a $\delta$-Jordan Lie supertriple system; then the following statements hold:

(1) $G \operatorname{Der}(T), Q \operatorname{Der}(T)$, and $C(T)$ are subalgebras of $\operatorname{End}(T)$.

(2) $Z \operatorname{Der}(T)$ is an ideal of $\operatorname{Der}(T)$.

Proof. (1) We only prove that $G \operatorname{Der}(T)$ is a subalgebra of $\operatorname{End}(T)$, and similarly for cases of $Q \operatorname{Der}(T)$ and $C(T)$. For any $D_{1} \in \operatorname{GDer}_{k}(T), D_{2} \in \operatorname{GDer}_{l}(T)$ and $a, b, c \in T$, we have

$$
\begin{aligned}
& {\left[D_{1} D_{2}(a), b, c\right]=\delta^{k} D_{1}^{\prime \prime \prime}\left[D_{2}(a), b, c\right]} \\
& -(-1)^{\left|D_{1}\right|\left(\left|D_{2}\right|+|a|\right)}\left[D_{2}(a), D_{1}^{\prime}(b), c\right] \\
& -(-1)^{\left|D_{1}\right|\left(\left|D_{2}\right|+|a|+|b|\right)}\left[D_{2}(a), b, D_{1}^{\prime \prime}(c)\right] \\
& =\delta^{k} D_{1}^{\prime \prime \prime}\left\{\delta^{l} D_{2}^{\prime \prime \prime}([a, b, c])\right. \\
& -(-1)^{\left|D_{2}\right||a|}\left[a, D_{2}^{\prime}(b), c\right] \\
& \left.-(-1)^{\left|D_{2}\right|(|a|+|b|)}\left[a, b, D_{2}^{\prime \prime}(c)\right]\right\}-(-1)^{\left|D_{1}\right|\left(\left|D_{2}\right|+|a|\right)} \\
& \cdot\left[D_{2}(a), D_{1}^{\prime}(b), c\right]-(-1)^{\left|D_{1}\right|\left(\left|D_{2}\right|+|a|+|b|\right)} \\
& \cdot\left[D_{2}(a), b, D_{1}^{\prime \prime}(c)\right]=\delta^{k+l} D_{1}^{\prime \prime \prime} D_{2}^{\prime \prime \prime}([a, b, c]) \\
& -\delta^{l}(-1)^{\left|D_{2}\right||a|} D_{1}^{\prime \prime \prime}\left[a, D_{2}^{\prime}(b), c\right]-\delta^{l}(-1)^{\left|D_{2}\right|(|a|+|b|)} \\
& \cdot D_{1}^{\prime \prime \prime}\left[a, b, D_{2}^{\prime \prime}(c)\right]-(-1)^{\left|D_{1}\right|\left(\left|D_{2}\right|+|a|\right)} \\
& \cdot\left[D_{2}(a), D_{1}^{\prime}(b), c\right]-(-1)^{\left|D_{1}\right|\left(\left|D_{2}\right|+|a|+|b|\right)} \\
& \cdot\left[D_{2}(a), b, D_{1}^{\prime \prime}(c)\right]=\delta^{k+l} D_{1}^{\prime \prime \prime} D_{2}^{\prime \prime \prime}([a, b, c]) \\
& -\delta^{k+l}(-1)^{\left|D_{2}\right||a|}\left[D_{1}(a), D_{2}^{\prime}(b), c\right] \\
& -\delta^{k+l}(-1)^{\left(\left|D_{1}\right|+\left|D_{2}\right|\right)|a|}\left[a, D_{1}^{\prime} D_{2}^{\prime}(b), c\right] \\
& -\delta^{k+l}(-1)^{\left|D_{2}\right||a|+\left|D_{1}\right|\left(|a|+|b|+\left|D_{2}\right|\right)}\left[a, D_{2}^{\prime}(b), D_{1}^{\prime \prime}(c)\right] \\
& -\delta^{k+l}(-1)^{\left|D_{2}\right|(|a|+|b|)}\left[D_{1}(a), b, D_{2}^{\prime \prime}(c)\right] \\
& -\delta^{k+l}(-1)^{\left|D_{2}\right|(|a|+|b|)+\left|D_{1}\right||a|}\left[a, D_{1}^{\prime}(b), D_{2}^{\prime \prime}(c)\right] \\
& -\delta^{k+l}(-1)^{\left(\left|D_{1}\right|+\left|D_{2}\right|\right)(|a|+|b|)}\left[a, b, D_{1}^{\prime \prime} D_{2}^{\prime \prime}(c)\right] \\
& -(-1)^{\left|D_{1}\right|\left(\left|D_{2}\right|+|a|\right)}\left[D_{2}(a), D_{1}^{\prime}(b), c\right] \\
& -(-1)^{\left|D_{1}\right|\left(\left|D_{2}\right|+|a|+|b|\right)}\left[D_{2}(a), b, D_{1}^{\prime \prime}(c)\right] .
\end{aligned}
$$

Similarly, we have

$$
\begin{aligned}
{[} & \left.D_{2} D_{1}(a), b, c\right] \\
= & \delta^{k+l} D_{2}^{\prime \prime \prime} D_{1}^{\prime \prime \prime}([a, b, c]) \\
& -\delta^{k+l}(-1)^{\left|D_{1}\right||a|}\left[D_{2}(a), D_{1}^{\prime}(b), c\right] \\
& -\delta^{k+l}(-1)^{\left(\left|D_{1}\right|+\left|D_{2}\right|\right)|a|}\left[a, D_{2}^{\prime} D_{1}^{\prime}(b), c\right] \\
& -\delta^{k+l}(-1)^{\left|D_{1}\right||a|+\left|D_{2}\right|\left(|a|+|b|+\left|D_{1}\right|\right)}\left[a, D_{1}^{\prime}(b), D_{2}^{\prime \prime}(c)\right] \\
& -\delta^{k+l}(-1)^{\left|D_{1}\right|(|a|+|b|)}\left[D_{2}(a), b, D_{1}^{\prime \prime}(c)\right] \\
& -\delta^{k+l}(-1)^{\left|D_{1}\right|(|a|+|b|)+\left|D_{2}\right||a|}\left[a, D_{2}^{\prime}(b), D_{1}^{\prime \prime}(c)\right] \\
& -\delta^{k+l}(-1)^{\left(\left|D_{1}\right|+\left|D_{2}\right|\right)(|a|+|b|)}\left[a, b, D_{2}^{\prime \prime} D_{1}^{\prime \prime}(c)\right]
\end{aligned}
$$




$$
\begin{aligned}
& -(-1)^{\left|D_{2}\right|\left(\left|D_{1}\right|+|a|\right)}\left[D_{1}(a), D_{2}^{\prime}(b), c\right] \\
& -(-1)^{\left|D_{2}\right|\left(\left|D_{1}\right|+|a|+|b|\right)}\left[D_{1}(a), b, D_{2}^{\prime \prime}(c)\right] .
\end{aligned}
$$

It follows that

$$
\begin{aligned}
{[} & {\left.\left[D_{1}, D_{2}\right](a), b, c\right]=\left[D_{1} D_{2}(a), b, c\right]-(-1)^{k+l} } \\
\cdot & {\left[D_{2} D_{1}(a), b, c\right] } \\
& =\delta^{k+l}\left(D_{1}^{\prime \prime \prime} D_{2}^{\prime \prime \prime}-(-1)^{\left|D_{1}\right|+\left|D_{2}\right|} D_{2}^{\prime \prime \prime} D_{1}^{\prime \prime \prime}\right)[a, b, c] \\
& -(-1)^{|a|\left(\left|D_{1}\right|+\left|D_{2}\right|\right)} \\
& \cdot\left[a,\left(D_{1}^{\prime} D_{2}^{\prime}-(-1)^{\left|D_{1}\right|+\left|D_{2}\right|} D_{2}^{\prime} D_{1}^{\prime}\right)(b), c\right] \\
& -(-1)^{(|a|+|b|)\left(\left|D_{1}\right|+\left|D_{2}\right|\right)} \\
& \cdot\left[a, b,\left(D_{1}^{\prime \prime} D_{2}^{\prime \prime}-(-1)^{\left|D_{1}\right|+\left|D_{2}\right|} D_{2}^{\prime \prime} D_{1}^{\prime \prime}\right)(c)\right] \\
& =\delta^{k+l}\left[D_{1}^{\prime \prime \prime}, D_{2}^{\prime \prime \prime}, a, b, c\right]-(-1)^{|a|\left(\left|D_{1}\right|+\left|D_{2}\right|\right)} \\
& \cdot\left[a,\left[D_{1}^{\prime}, D_{2}^{\prime}\right](b), c\right]-(-1)^{(|a|+|b|)\left(\left|D_{1}\right|+\left|D_{2}\right|\right)} \\
& \cdot\left[a, b,\left[D_{1}^{\prime \prime}, D_{2}^{\prime \prime}\right](c)\right] .
\end{aligned}
$$

Obviously, $\left[D_{1}^{\prime}, D_{2}^{\prime}\right],\left[D_{1}^{\prime \prime}, D_{2}^{\prime \prime}\right]$ and $\left[D_{1}^{\prime \prime \prime}, D_{2}^{\prime \prime \prime}\right]$ are contained in $\operatorname{End}(T)$; thus $\left[D_{1}, D_{2}\right] \in \operatorname{GDer}_{k+l}(T) \subseteq G \operatorname{Der}(T)$; that is, $\operatorname{GDer}(T)$ is a subalgebra of $\operatorname{End}(T)$.

(2) For any $D_{1} \in Z \operatorname{Der}(T), D_{2} \in \operatorname{Der}_{k}(T)$, and $a, b, c \in T$, we have

$$
\begin{aligned}
{\left[D_{1}, D_{2}\right]([a, b, c])=} & D_{1} D_{2}([a, b, c]) \\
& -(-1)^{\left|D_{1}\right|\left|D_{2}\right|} D_{2} D_{1}([a, b, c]) \\
= & 0 .
\end{aligned}
$$

Also, we have

$$
\begin{aligned}
& {\left[\left[D_{1}, D_{2}\right](a), b, c\right]=\left[D_{1} D_{2}(a), b, c\right]-(-1)^{\left|D_{1}\right|\left|D_{2}\right|}} \\
& \cdot\left[D_{2} D_{1}(a), b, c\right]=0-(-1)^{\left|D_{1}\right|\left|D_{2}\right|} \\
& \cdot\left[D_{2} D_{1}(a), b, c\right]=-(-1)^{\left|D_{1}\right|\left|D_{2}\right|} \\
& \cdot\left(\delta^{k} D_{2}\left(\left[D_{1}(a), b, c\right]\right)\right. \\
& \left.-(-1)^{\left|D_{2}\right|\left(\left|D_{1}\right|+|a|\right)}\left[D_{1}(a), D_{2}(b), c\right]\right) \\
& +(-1)^{\left|D_{1}\right|\left|D_{2}\right|}(-1)^{\left|D_{2}\right|\left(\left|D_{1}\right|+|a|+|b|\right)}\left[D_{1}(a), b, D_{2}(c)\right] \\
& \quad=0
\end{aligned}
$$

It follows that $\left[D_{1}, D_{2}\right] \in Z \operatorname{Der}(T)$. That is, $Z \operatorname{Der}(T)$ is an ideal of $\operatorname{Der}(T)$.

Proposition 15. Let $T$ be a $\delta$-Jordan Lie supertriple system; then the following statements hold:
(1) $[\operatorname{Der}(T), C(T)] \subseteq C(T)$.
(2) $[Q \operatorname{Der}(T), Q C(T)] \subseteq Q C(T)$.
(3) $[Q C(T), Q C(T)] \subseteq Q \operatorname{Der}(T)$.
(4) $C(T) \subseteq Q \operatorname{Der}(T)$.

Proof. (1) For any $D_{1} \in \operatorname{Der}_{k}(T), D_{2} \in C_{l}(T)$, and $a, b, c \in T$, we have

$$
\begin{aligned}
{\left[D_{1}, D_{2}\right]([a, b, c]) } & D_{1} D_{2}([a, b, c])-(-1)^{\left|D_{1}\right|\left|D_{2}\right|} D_{2} D_{1}([a, b, c]) \\
= & \delta^{l} D_{1}\left(\left[D_{2}(a), b, c\right]\right) \\
& -\delta^{k}(-1)^{\left|D_{1}\right|\left|D_{2}\right|} D_{2}\left(\left[D_{1}(a), b, c\right]\right) \\
& -\delta^{k}(-1)^{\left|D_{1}\right|\left(\left|D_{2}\right|+|a|\right)} D_{2}\left(\left[a, D_{1}(b), c\right]\right) \\
& -\delta^{k}(-1)^{\left|D_{1}\right|\left(\left|D_{2}\right|+|a|+|b|\right)} D_{2}\left(\left[a, b, D_{1}(c)\right]\right) \\
= & \delta^{k+l}\left[D_{1} D_{2}(a), b, c\right] \\
& +\delta^{k+l}(-1)^{\left|D_{1}\right|\left(\left|D_{2}\right|+|a|\right)}\left[D_{2}(a), D_{1}(b), c\right] \\
& +\delta^{k+l}(-1)^{\left|D_{1}\right|\left(\left|D_{2}\right|+|a|+|b|\right)}\left[D_{2}(a), b, D_{1}(c)\right] \\
& -\delta^{k+l}(-1)^{\left|D_{1}\right|\left|D_{2}\right|}\left[D_{2} D_{1}(a), b, c\right] \\
& -\delta^{k+l}(-1)^{\left|D_{1}\right|\left(\left|D_{2}\right|+|a|\right)}\left[D_{2}(a), D_{1}(b), c\right] \\
& -\delta^{k+l}(-1)^{\left|D_{1}\right|\left(\left|D_{2}\right|+|a|+|b|\right)}\left[D_{2}(a), b, D_{1}(c)\right] \\
= & \delta^{k+l}\left[\left[D_{1}, D_{2}\right](a), b, c\right] .
\end{aligned}
$$

Similarly, one can check that

$$
\begin{aligned}
& {\left[\left[D_{1}, D_{2}\right](a), b, c\right]=(-1)^{|a|(k+l)}\left[a,\left[D_{1}, D_{2}\right](b), c\right]} \\
& \quad=(-1)^{(|a|+|b|)(k+l)}\left[a, b,\left[D_{1}, D_{2}\right](c)\right] .
\end{aligned}
$$

It follows that $\left[D_{1}, D_{2}\right] \in C(T)$; thus $[\operatorname{Der}(T), C(T)] \subseteq C(T)$.

(2) Similar to the proof of (1).

(3) For any $D_{1}, D_{2} \in Q C(T)$ and $a, b, c \in T$, we have

$$
\begin{aligned}
{\left[\left[D_{1}, D_{2}\right](a), b, c\right] } & +(-1)^{|a|\left(\left|D_{1}\right|+\left|D_{2}\right|\right)}\left[a,\left[D_{1}, D_{2}\right](b), c\right] \\
& +(-1)^{(|a|+|b|)\left(\left|D_{1}\right|+\left|D_{2}\right|\right)}\left[a, b,\left[D_{1}, D_{2}\right](c)\right] \\
= & {\left[D_{1} D_{2}(a), b, c\right] } \\
& +(-1)^{|a|\left(\left|D_{1}\right|+\left|D_{2}\right|\right)}\left[a, D_{1} D_{2}(b), c\right] \\
& +(-1)^{(|a|+|b|)\left(\left|D_{1}\right|+\left|D_{2}\right|\right)}\left[a, b, D_{1} D_{2}(c)\right] \\
& -(-1)^{\left|D_{1}\right|\left|D_{2}\right|}\left[D_{2} D_{1}(a), b, c\right] \\
& -(-1)^{|a|\left(\left|D_{1}\right|+\left|D_{2}\right|\right)+\left|D_{1}\right|\left|D_{2}\right|}\left[a, D_{2} D_{1}(b), c\right] \\
& -(-1)^{(|a|+|b|)\left(\left|D_{1}\right|+\left|D_{2}\right|\right)+\left|D_{1}\right|\left|D_{2}\right|}\left[a, b, D_{2} D_{1}(c)\right] .
\end{aligned}
$$


Since $D_{1}, D_{2} \in Q C(T)$, we have

$$
\begin{gathered}
{\left[D_{1} D_{2}(a), b, c\right]=(-1)^{\left|D_{1}\right|\left(|a|+\left|D_{2}\right|\right)}\left[D_{2}(a), D_{1}(b), c\right]} \\
=(-1)^{\left|D_{1}\right|\left(|a|+\left|D_{2}\right|\right)+|a|\left|D_{2}\right|}\left[a, D_{2} D_{1}(b), c\right]
\end{gathered}
$$

Similarly, one may check that

$$
\begin{aligned}
& {\left[a, D_{1} D_{2}(b), c\right]} \\
& \quad=(-1)^{|b|\left(\left|D_{1}\right|+\left|D_{2}\right|\right)+\left|D_{1}\right|\left|D_{2}\right|}\left[a, b, D_{2} D_{1}(c)\right], \\
& {\left[a, b, D_{1} D_{2}(c)\right]} \\
& \quad=(-1)^{(|a|+|b|)\left(\left|D_{1}\right|+\left|D_{2}\right|\right)+\left|D_{1}\right|\left|D_{2}\right|}\left[D_{2} D_{1}(a), b, c\right] .
\end{aligned}
$$

It follows that

$$
\begin{aligned}
& {\left[\left[D_{1}, D_{2}\right](a), b, c\right]} \\
& \quad+(-1)^{|a|\left(\left|D_{1}\right|+\left|D_{2}\right|\right)}\left[a,\left[D_{1}, D_{2}\right](b), c\right] \\
& \quad+(-1)^{(|a|+|b|)\left(\left|D_{1}\right|+\left|D_{2}\right|\right)}\left[a, b,\left[D_{1}, D_{2}\right](c)\right]=0 .
\end{aligned}
$$

Therefore, $\left[D_{1}, D_{2}\right] \subseteq Q \operatorname{Der}(T)$ and $[Q C(T), Q C(T)] \subseteq$ $\mathrm{QDer}(T)$.

(4) For any $D \in C_{k}(T)$ and $a, b, c \in T$, we have

$$
\begin{aligned}
D([a, b, c]) & =\delta^{k}[D(a), b, c] \\
& =\delta^{k}(-1)^{|D||a|}[a, D(b), c] \\
& =\delta^{k}(-1)^{|D|(|a|+|b|)}[a, b, D(c)] .
\end{aligned}
$$

Thus

$$
\begin{aligned}
\delta^{k}[D & (a), b, c]+\delta^{k}(-1)^{|D||a|}[a, D(b), c] \\
& +\delta^{k}(-1)^{|D|(|a|+|b|)}[a, b, D(c)]=3 D([a, b, c]),
\end{aligned}
$$

that is, $D \in \operatorname{QDer}_{k}(T)$ and $Q C(T) \subseteq Q \operatorname{Der}(T)$.

Theorem 16. Let $T$ be a $\delta$-Jordan Lie supertriple system; then $[C(T), Q C(T)] \subseteq \operatorname{End}(T, Z(T))$. Moreover, if $Z(T)=\{0\}$, then $[C(T), Q C(T)]=\{0\}$.

Proof. For any $D_{1} \in C_{k}(T), D_{2} \in Q C_{l}(T)$, and $a, b, c \in T$, we have

$$
\begin{aligned}
{[} & {\left.\left[D_{1}, D_{2}\right](a), b, c\right] } \\
= & {\left[D_{1} D_{2}(a), b, c\right]-(-1)^{\left|D_{1}\right|\left|D_{2}\right|}\left[D_{2} D_{1}(a), b, c\right] } \\
= & \delta^{k} D_{1}\left(\left[D_{2}(a), b, c\right]\right) \\
& -(-1)^{\left|D_{1}\right|\left|D_{2}\right|}(-1)^{\left(\left|D_{1}\right|+|a|\right)\left|D_{2}\right|}\left[D_{1}(a), D_{2}(b), c\right] \\
= & \delta^{k}(-1)^{|a|\left|D_{2}\right|} D_{1}\left(\left[a, D_{2}(b), c\right]\right) \\
& -(-1)^{|a|\left|D_{2}\right|} D_{1}\left(\left[a, D_{2}(b), c\right]\right)=0 .
\end{aligned}
$$

So $\left[D_{1}, D_{2}\right](a) \subseteq Z(T)$ and therefore $[C(T), Q C(T)] \subseteq$ $\operatorname{End}(T, Z(T))$. Moreover, if $Z(T)=\{0\}$, then it is easy to see that $[C(T), Q C(T)]=\{0\}$.
Theorem 17. Let $T$ be a $\delta$-Jordan Lie supertriple system and Char $k \neq 2$; then $Z \operatorname{Dert}=C(T) \cap \operatorname{Der}(T)$.

Proof. For any $D \in C_{k}(T) \cap \operatorname{Der}_{k}(T)$ and $a, b, c \in T$, we have

$$
\begin{aligned}
D([a, b, c])= & \delta^{k}[D(a), b, c] \\
& +\delta^{k}(-1)^{|D||a|}[a, D(b), c] \\
& +\delta^{k}(-1)^{|D|(|a|+|b|)}[a, b, D(c)], \\
D([a, b, c])= & \delta^{k}[D(a), b, c] \\
= & \delta^{k}(-1)^{|D||a|}[a, D(b), c] \\
= & \delta^{k}(-1)^{|D|(|a|+|b|)}[a, b, D(c)] .
\end{aligned}
$$

It follows that $2 D([a, b, c])=0$. Thus $D([a, b, c])=0$ since Char $k \neq 2$; that is, $D \in Z \operatorname{Dert}$. So $C(T) \cap \operatorname{Der}(T) \in Z \operatorname{Dert}$.

On the other hand, for any $D \in Z \operatorname{Dert}$ and $a, b, c \in T$, we have $D([a, b, c])=0$. Clearly, (6) and (11) hold; that is, $D \in \mathrm{C}_{k}(T) \cap \operatorname{Der}_{k}(T)$, and, therefore, $Z \operatorname{Dert} \in C(T) \cap \operatorname{Der}(T)$. And this completes the proof.

\section{The Cohomology of $\delta$-Jordan Lie Supertriple Systems}

In this section, we introduce the notion of the representation of $\delta$-Jordan Lie supertriple systems and present its lowdimensional cohomologies.

Definition 18. Let $T$ be a $\delta$-Jordan Lie supertriple system and $V$ a $Z_{2}$-graded vector space. Suppose that there exists a bilinear mapping $\theta: T \otimes T \longrightarrow \operatorname{End}(V)$ satisfying the following axioms:

$$
\begin{aligned}
(-1)^{(|a|+|b|)(|c|+|d|)} \theta(c, d) \theta(a, b) \\
\quad-\delta(-1)^{(|a||b|+|d|(|c|+|a|))} \theta(b, d) \theta(a, c) \\
\quad-\theta(a,[b, c, d]) \\
\quad+(-1)^{|a|(|b|+|c|)} D(b, c) \theta(a, d)=0, \\
\delta(-1)^{(|a|+|b|)(|c|+|d|)} \theta(c, d) D(a, b)-\delta D(a, b) \theta(c, d) \\
\quad+\theta([a, b, c], d) \\
\quad+\delta(-1)^{|c| \mid(|a|+|b|)} \theta(c,[a, b, d])=0, \\
D([a, b, c], d)+(-1)^{|c|(|a|+|b|)} D(c,[a, b, d]) \\
\quad-\delta D(a, b) D(c, d) \\
\quad+(-1)^{(|a|+|b|)(|c|+|d|)} D(c, d) D(a, b),
\end{aligned}
$$

for $a, b, c, d \in T$, where $D(a, b)=(-1)^{|a||b|} \theta(b, a)-\delta \theta(a, b)$; then $(V, \theta)$ is called the representation of $T ; V$ is called a $T$-module. 
Example 19. Let $T$ be a $\delta$-Jordan Lie supertriple system. Define $\theta: T \otimes T \longrightarrow \operatorname{End}(T)$ by $\theta(a, b)(x)=$ $(-1)^{|x|(|a|+|b|)}[x, a, b]$. It is not hard to check that $D(a, b)(x)=$ $\delta[a, b, x]$ and $T$ itself is a $T$-module. In this case, $(T, \theta)$ is said to be the adjoint representation of $T$.

Proposition 20. Let $T$ be a $\delta$-Jordan Lie supertriple system and $(V, \theta)$ the representation. Then $T \oplus V$ has a structure of a $\delta$-Jordan Lie supertriple system.

Proof. Define a triple linear product $[\cdot, \cdot, \cdot]:(T \oplus V) \otimes(T \oplus$ $V) \otimes(T \oplus V) \longrightarrow(T \oplus V)$ by

$$
\begin{aligned}
& {[(a, u),(b, v),(c, w)]} \\
& \quad=\left([a, b, c],(-1)^{|a|(|b|+|c|)} \theta(b, c)(u)\right. \\
& \left.\quad-\delta(-1)^{|b||c|} \theta(a, c)(v)+\delta D(a, b)(w)\right),
\end{aligned}
$$

for all $(a, u),(b, v),(c, w) \in T \oplus V$, where $|a, u|=|a|$.

Now we check that the operation $[\cdot, \cdot, \cdot]$ defined above satisfies axioms in Definition 1 . It is easy to see that (1) holds since $T$ is a $\delta$-Jordan Lie supertriple system.

For (2), we take any $(a, u),(b, v),(c, w) \in T \oplus V$ and compute

$$
\begin{aligned}
- & \delta(-1)^{|a||b|}[(b, v),(a, u),(c, w)]=-\delta(-1)^{|a||b|} \\
\cdot & \left([b, a, c],(-1)^{|b|(|a|+|c|)} \theta(a, c)(v)\right. \\
- & \left.\delta(-1)^{|a||c|} \theta(b, c)(u)+\delta D(b, a)(w)\right) \\
= & \left(-\delta(-1)^{|a||b|}[b, a, c],-\delta(-1)^{|b||c|} \theta(a, c)(v)\right. \\
- & (-1)^{|a|(|b|+|c|)} \theta(b, c)(u) \\
- & \left.\delta(-1)^{|a||b|} \delta D(b, a)(w)\right) \\
= & \left([a, b, c],(-1)^{|a|(|b|+|c|)} \theta(b, c)(u)\right. \\
- & \left.\delta(-1)^{|b||c|} \theta(a, c)(v)+\delta D(a, b)(w)\right) \\
= & {[(a, u),(b, v),(c, w)] . }
\end{aligned}
$$

The last equality holds since $-(-1)^{|a||b|}[b, a, w]=[a, b, w]=$ $\delta D(a, b)(w)$.

For (3), we have

$$
\begin{aligned}
(-1)^{|a||c|}[(a, u),(b, v),(c, w)]+(-1)^{|b||a|} \\
\cdot[(b, v),(c, w),(a, u)]+(-1)^{|c||b|} \\
\cdot[(c, w),(a, u),(b, v)]=\left((-1)^{|a||c|}[a, b, c]\right. \\
\left.+(-1)^{|b||a|}[b, c, a]+(-1)^{|c||b|}[c, a, b], \Omega\right)=(0, \Omega),
\end{aligned}
$$

where

$$
\begin{aligned}
\Omega= & (-1)^{|a||b|} \theta(b, c)(u)-\delta(-1)^{(|a|+|b|)|c|} \theta(a, c)(v) \\
& +\delta(-1)^{|a||c|} D(a, b)(w)+(-1)^{|b||c|} \theta(c, a)(v) \\
& -\delta(-1)^{(|b|+|c|)|a|} \theta(b, a)(w) \\
& +\delta(-1)^{|b||a|} D(b, c)(u)+(-1)^{|c||b|} \theta(a, b)(w) \\
& -\delta(-1)^{(|c|+|a|)|b|} \theta(c, b)(u) \\
& +\delta(-1)^{|c||b|} D(c, a)(v) \\
= & (-1)^{|a||b|} \theta(b, c)(u)+(-1)^{|b||c|} \theta(c, a)(v) \\
& +(-1)^{|c||b|} \theta(a, b)(w)-(-1)^{|a||c|} \theta(a, b)(w) \\
& +(-1)^{|b||a|} \theta(b, c)(u)-(-1)^{|b||c|} \theta(c, a)(v)=0 .
\end{aligned}
$$

The second equality holds since $D(a, b)=(-1)^{|a||b|} \theta(b, a)-$ $\delta \theta(a, b)$. Then we have

$$
\begin{aligned}
& (-1)^{|a||c|}[(a, u),(b, v),(c, w)] \\
& \quad+(-1)^{|b||a|}[(b, v),(c, w),(a, u)] \\
& \quad+(-1)^{|c||b|}[(c, w),(a, u),(b, v)]=(0,0),
\end{aligned}
$$

as desired.

For $(4)$, we take any $(a, u),(b, v),(c, w),(d, m),(e, n) \in T \oplus$ $V$. First, we calculate the following expression:

$$
\begin{aligned}
& {[[(a, u),(b, v),(c, w)],(d, m),(e, n)]} \\
& \quad=\left[\left([a, b, c],(-1)^{|a|(|b|+|c|)} \theta(b, c)(u)\right.\right. \\
& \left.\quad-\delta(-1)^{|b||c|} \theta(a, c)(v)+\delta D(a, b)(w)\right),(d, m), \\
& \quad(e, n)]=\left([[a, b, c], d, e], \Omega_{1}\right),
\end{aligned}
$$

where

$$
\begin{aligned}
\Omega_{1} & \\
= & (-1)^{(|a|+|b|+|c|)(|d|+|e|)}(-1)^{|a|(|b|+|c|)} \theta(d, e) \theta(b, c)(u) \\
& -\delta(-1)^{(|a|+|b|+|c|)(|d|+|e|)}(-1)^{|b||c|} \theta(d, e) \theta(a, c)(v) \\
& +\delta(-1)^{(|a|+|b|+|c|)(|d|+|e|)} \theta(d, e) D(a, b)(w) \\
& -\delta(-1)^{|d||e|} \theta([a, b, c], e)(m) \\
& +\delta D([a, b, c], d)(n) .
\end{aligned}
$$

Second, we compute the expression $(-1)^{|c|(|a|+|b|)}[(c$, $w),[(a, u),(b, v),(d, m)],(e, n)]$ : 


$$
\begin{aligned}
(-1)^{|c|(|a|+|b|)}[(c, w),[(a, u),(b, v),(d, m)],(e, n)] \\
=(-1)^{|c|(|a|+|b|)}[(c, w), \\
\left([a, b, d],(-1)^{|a|(|b|+|d|)} \theta(b, d)(u)\right. \\
\left.\left.-\delta(-1)^{|b||d|} \theta(a, d)(v)+\delta D(a, b)(m)\right),(e, n)\right] \\
=\left((-1)^{|c||| a|+| b \mid)}[c,[a, b, d], e], \Omega_{2}\right),
\end{aligned}
$$

where

$$
\begin{aligned}
\Omega_{2}=- & \delta(-1)^{(|a|+|b|+|d|)|e|}(-1)^{|c||(|a|+|b|)+| a \mid(|b|+|d|)} \theta(c, e) \\
\cdot & \theta(b, d)(u)+(-1)^{(|a|+|b|+|d|)|e|} \\
& \cdot(-1)^{|c||| a|+| b \mid)+|b||d|} \theta(c, e) \theta(a, d)(v) \\
+ & (-1)^{(|a|+|b|+|d|+|e|)|c|}(-1)^{|c|(|a|+|b|)} \theta([a, b, d], e) \\
& \cdot(w)-(-1)^{(|a|+|b|+|d|)|e|}(-1)^{|c||| a|+| b \mid)} \theta(c, e) \\
& \cdot D(a, b)(m)+\delta(-1)^{|c|(|a|+|b|)} D(c,[a, b, d]) \\
& \cdot(n) .
\end{aligned}
$$

Third, we compute the expression $\delta(-1)^{(|a|+|b|)(|c|+|d|)}[(c, w)$, $(d, m),[(a, u),(b, v),(e, n)]]$ :

$$
\begin{aligned}
\delta( & -1)^{(|a|+|b|)(|c|+|d|)}[(c, w),(d, m), \\
& {[(a, u),(b, v),(e, n)]]=\delta(-1)^{(|a|+|b|)(|c|+|d|)}[(c, w),} \\
& (d, m),\left([a, b, e],(-1)^{|a|(|b|+|e|)} \theta(b, e)(u)\right. \\
- & \left.\left.\delta(-1)^{|b||e|} \theta(a, e)(v)+\delta D(a, b)(n)\right)\right] \\
& =\left(\delta(-1)^{(|a|+|b|)(|c|+|d|)}[c, d,[a, b, e]], \Omega_{3}\right),
\end{aligned}
$$

where

$$
\begin{aligned}
\Omega_{3}= & -1)^{(|a|+|b|)(|c|+|d|)}(-1)^{|a|(|b|+|e|)} D(c, d) \theta(b, e)(u) \\
- & \delta(-1)^{(|a|+|b|)(|c|+|d|)}(-1)^{|b| e \mid} D(c, d) \theta(a, e)(v) \\
+ & \delta(-1)^{(|a|+|b|)(|c|+|d|)}(-1)^{(|a|+|b|+|d|+|e|)|c|} \\
& \cdot \theta(d,[a, b, e])(w)-(-1)^{(|a|+|b|)(|c|+|d|)} \\
& \cdot(-1)^{(|a|+|b|+|e|)|d|} \theta(c,[a, b, e])(m) \\
+ & \delta(-1)^{(|a|+|b|)(|c|+|d|)} D(c, d) D(a, b)(n) .
\end{aligned}
$$

Fourth, we compute the expression $[(a, u),(b, v),[(c, w)$, $(d, m),(e, n)]]$ :

$$
\begin{aligned}
& \quad[(a, u),(b, v),[(c, w),(d, m),(e, n)]]=[(a, u),(b, v), \\
& \quad\left([c, d, e],(-1)^{|c| \mid(d||+|e|)} \theta(d, e)(w)\right. \\
& \left.\left.\quad-\delta(-1)^{|d||| e \mid} \theta(c, e)(m)+\delta D(c, d)(n)\right)\right] \\
& \quad=\left([a, b,[c, d, e]], \Omega_{4}\right)
\end{aligned}
$$

where

$$
\begin{aligned}
\Omega_{4}= & (-1)^{|a|(|b|+|c|+|d|+|e|)} \theta(b,[c, d, e])(u) \\
& -\delta(-1)^{|b|(|c|+|d|+|e|)} \theta(a,[c, d, e])(v) \\
& +\delta(-1)^{|c|(|d|+|e|)} D(a, b) \theta(d, e)(w) \\
& -(-1)^{|d||e|} D(a, b) \theta(c, e)(m) \\
& +D(a, b) D(c, d)(n) .
\end{aligned}
$$

Finally, by (33), (34), and (35), we have

$$
\begin{aligned}
& {[[(a, u),(b, v),(c, w)],(d, m),(e, n)]+(-1)^{|c| \mid(|a|+|b|)}} \\
& \quad \cdot[(c, w),[(a, u),(b, v),(d, m)],(e, n)] \\
& \quad+\delta(-1)^{(|a|+|b|)(|c|+|d|)} \\
& \quad \cdot[(c, w),(d, m),[(a, u),(b, v),(e, n)]] \\
& \quad=\left([[a, b, c], d, e]+(-1)^{|c||| a|+| b \mid)}[c,[a, b, d], e]\right. \\
& \quad+\delta(-1)^{(|a|+|b|)(|c|+|d|)}[c, d,[a, b, e]], \Omega_{1}+\Omega_{2} \\
& \left.\quad+\Omega_{3}\right)=\left([a, b,[c, d, e]], \Omega_{4}\right) \\
& \quad=[(a, u),(b, v),[(c, w),(d, m),(e, n)]]
\end{aligned}
$$

as desired, and this finishes the proof.

Corollary 21. Any $\delta$-Jordan Lie supertriple system can be considered as a subspace of a $\delta$-Jordan Lie superalgebra.

Proof. It is straightforward from Example 19 and Proposition 20.

Definition 22. Let $T$ be a $\delta$-Jordan Lie supertriple system and $V$ a $T$-module by a bilinear map $\theta$. If an $n$-linear map $f: T \times$ $T \times \cdots \times T \longrightarrow T$ satisfies the following axioms

$$
\text { (1) } \begin{aligned}
& f\left(x_{1}, x_{2}, \ldots, x, y, \ldots, x_{n}\right) \\
& -\delta(-1)^{|x||y|} f\left(x_{1}, x_{2}, \ldots, x, y, \ldots, x_{n}\right) ; \\
\text { (2) } & (-1)^{|x||z|} f\left(x_{1}, x_{2}, \ldots, x_{n-3}, x, y, z\right) \\
& (-1)^{|y||x|} f\left(x_{1}, x_{2}, \ldots, x_{n-3}, y, z, x\right) \\
& (-1)^{|z| y \mid} f\left(x_{1}, x_{2}, \ldots, x_{n-3}, z, x, y\right)=0,
\end{aligned}
$$

then $f$ is called an $n$-cochain on $T$. Denote by $C_{\delta}^{n}(T, V)$ the set of all $n$-cochains, for $n \geq 1$.

Definition 23. Let $T$ be a $\delta$-Jordan Lie supertriple system and $V$ a $T$-module by a bilinear map $\theta$. For $n=1,2$, the coboundary operator $d_{n}: C_{\delta}^{n}(T, V) \longrightarrow C_{\delta}^{n+2}(T, V)$ is defined as follows:

(i) If $f \in C_{\delta}^{1}(T, V)$, then

$$
\begin{aligned}
d^{1} f & \left(x_{1}, x_{2}, x_{3}\right) \\
= & (-1)^{\left(|f|+\left|x_{1}\right|\right)\left(\left|x_{2}\right|+\left|x_{3}\right|\right)} \theta\left(x_{2}, x_{3}\right) f\left(x_{1}\right) \\
& -f\left(\left[x_{1}, x_{2}, x_{3}\right]\right)
\end{aligned}
$$




$$
\begin{aligned}
& +\delta(-1)^{|f|\left(\left|x_{1}\right|+\left|x_{2}\right|\right)} D\left(x_{1}, x_{2}\right) f\left(x_{3}\right) \\
& -\delta(-1)^{\left|x_{2}\right|\left|x_{3}\right|+|f|\left(\left|x_{1}\right|+\left|x_{3}\right|\right)} \theta\left(x_{1}, x_{3}\right) f\left(x_{2}\right) .
\end{aligned}
$$

(ii) If $f \in C_{\delta}^{2}(T, V)$, then

$$
\begin{aligned}
d^{2} f & \left(y, x_{1}, x_{2}, x_{3}\right) \\
= & (-1)^{\left(|f|+|y|+\left|x_{1}\right|\right)\left(\left|x_{2}\right|+\left|x_{3}\right|\right)} \theta\left(x_{2}, x_{3}\right) f\left(y, x_{1}\right) \\
& -f\left(y,\left[x_{1}, x_{2}, x_{3}\right]\right) \\
& -\delta(-1)^{\left|x_{2}\right|\left|x_{3}\right|+\left(|f|+\left|y_{1}\right|\right)\left(\left|x_{1}\right|+\left|x_{3}\right|\right)} \theta\left(x_{1}, x_{3}\right) f\left(y, x_{2}\right) \\
& +\delta(-1)^{(|f|+|y|)\left(\left|x_{1}\right|+\left|x_{2}\right|\right)} D\left(x_{1}, x_{2}\right) f\left(y, x_{3}\right) .
\end{aligned}
$$

(iii) If $f \in C_{\delta}^{3}(T, V)$, then

$$
\begin{array}{rl}
d^{3} & f\left(x_{1}, x_{2}, x_{3}, x_{4}, x_{5}\right)=(-1)^{\left(|f|+\left|x_{1}\right|+\left|x_{2}\right|+\left|x_{3}\right|\right)\left(\left|x_{4}\right|+\left|x_{5}\right|\right)} \\
& \cdot \theta\left(x_{4}, x_{5}\right) f\left(x_{1}, x_{2}, x_{3}\right) \\
& -\delta(-1)^{\left(|f|+\left|x_{1}\right|+\left|x_{2}\right|\right)\left(\left|x_{3}\right|+\left|x_{5}\right|\right)+\left|x_{4}\right|\left|x_{5}\right|} \theta\left(x_{3}, x_{5}\right) \\
& \cdot f\left(x_{1}, x_{2}, x_{4}\right)-\delta(-1)^{|f|\left(\left|x_{1}\right|+\left|x_{2}\right|\right)} D\left(x_{1}, x_{2}\right) \\
& \cdot f\left(x_{3}, x_{4}, x_{5}\right)+(-1)^{\left(|f|+\left|x_{1}\right|+\left|x_{2}\right|\right)\left(\left|x_{3}\right|+\left|x_{4}\right|\right)} \\
& \cdot D\left(x_{3}, x_{4}\right) f\left(x_{1}, x_{2}, x_{5}\right)+f\left(\left[x_{1}, x_{2}, x_{3}\right], x_{4}, x_{5}\right) \\
& -f\left(x_{1}, x_{2},\left[x_{3}, x_{4}, x_{5}\right]\right)+(-1)^{\left|x_{3}\right|\left(\left|x_{1}\right|+\left|x_{2}\right|\right)} \\
& \cdot f\left(x_{3},\left[x_{1}, x_{2}, x_{4}\right], x_{5}\right)+\delta(-1)^{\left(\left|x_{1}\right|+\left|x_{2}\right|\right)\left(\left|x_{3}\right|+\left|x_{4}\right|\right)} \\
& \cdot f\left(x_{3}, x_{4},\left[x_{1}, x_{2}, x_{5}\right]\right) .
\end{array}
$$

(iv) If $f \in C_{\delta}^{4}(T, V)$, then

$$
\begin{array}{rl}
d^{4} & f\left(y, x_{1}, x_{2}, x_{3}, x_{4}, x_{5}\right) \\
= & (-1)^{\left(|f|+|y|+\left|x_{1}\right|+\left|x_{2}\right|+\left|x_{3}\right|\right)\left(\left|x_{4}\right|+\left|x_{5}\right|\right)} \theta\left(x_{4}, x_{5}\right) \\
& \cdot f\left(y, x_{1}, x_{2}, x_{3}\right) \\
& -\delta(-1)^{\left(|f|+|y|+\left|x_{1}\right|+\left|x_{2}\right|\right)\left(\left|x_{3}\right|+\left|x_{5}\right|\right)+\left|x_{4}\right|\left|x_{5}\right|} \theta\left(x_{3}, x_{5}\right) \\
& \cdot f\left(y, x_{1}, x_{2}, x_{4}\right)-\delta(-1)^{(|f|+|y|)\left(\left|x_{1}\right|+\left|x_{2}\right|\right)} \\
& \cdot D\left(x_{1}, x_{2}\right) f\left(y, x_{3}, x_{4}, x_{5}\right) \\
& +(-1)^{\left(|f|+|y|+\left|x_{1}\right|+\left|x_{2}\right|\right)\left(\left|x_{3}\right|+\left|x_{4}\right|\right)} D\left(x_{3}, x_{4}\right) \\
& \cdot f\left(y, x_{1}, x_{2}, x_{5}\right)+f\left(y,\left[x_{1}, x_{2}, x_{3}\right], x_{4}, x_{5}\right) \\
& -f\left(y, x_{1}, x_{2},\left[x_{3}, x_{4}, x_{5}\right]\right)+(-1)^{\left|x_{3}\right|\left(\left|x_{1}\right|+\left|x_{2}\right|\right)} \\
& \cdot f\left(y, x_{3},\left[x_{1}, x_{2}, x_{4}\right], x_{5}\right) \\
& +\delta(-1)^{\left(\left|x_{1}\right|+\left|x_{2}\right|\right)\left(\left|x_{3}\right|+\left|x_{4}\right|\right)} f\left(y, x_{3}, x_{4},\left[x_{1}, x_{2}, x_{5}\right]\right) .
\end{array}
$$

Theorem 24. Let $T$ be a $\delta$-Jordan Lie supertriple system and $V$ a T-module by a bilinear map $\theta$. The coboundary operator $d^{n}$ defined above satisfies $d^{n+2} d^{n}=0, n=1,2$.

Proof. From the definition of the coboundary operator, it follows immediately that $d^{3} d^{1}=0$ implies $d^{4} d^{2}=0$. Then, we only need to prove $d^{3} d^{1}=0$ :

$$
\begin{aligned}
d^{3} & \left(d^{1} f\right)\left(x_{1}, x_{2}, x_{3}, x_{4}, x_{5}\right) \\
= & (-1)^{\left(|f|+\left|x_{1}\right|+\left|x_{2}\right|+\left|x_{3}\right|\right)\left(\left|x_{4}\right|+\left|x_{5}\right|\right)} \theta\left(x_{4}, x_{5}\right)\left(d^{1} f\right) \\
& \cdot\left(x_{1}, x_{2}, x_{3}\right) \\
- & \delta(-1)^{\left(|f|+\left|x_{1}\right|+\left|x_{2}\right|\right)\left(\left|x_{3}\right|+\left|x_{5}\right|\right)+\left|x_{4}\right|\left|x_{5}\right|} \theta\left(x_{3}, x_{5}\right)\left(d^{1} f\right) \\
& \cdot\left(x_{1}, x_{2}, x_{4}\right) \\
- & \delta(-1)^{|f|\left(\left|x_{1}\right|+\left|x_{2}\right|\right)} D\left(x_{1}, x_{2}\right)\left(d^{1} f\right)\left(x_{3}, x_{4}, x_{5}\right) \\
+ & (-1)^{\left(|f|+\left|x_{1}\right|+\left|x_{2}\right|\right)\left(\left|x_{3}\right|+\left|x_{4}\right|\right)} D\left(x_{3}, x_{4}\right)\left(d^{1} f\right)\left(x_{1}, x_{2}, x_{5}\right) \\
+ & \left(d^{1} f\right)\left(\left[x_{1}, x_{2}, x_{3}\right], x_{4}, x_{5}\right)-\left(d^{1} f\right) \\
& \cdot\left(x_{1}, x_{2},\left[x_{3}, x_{4}, x_{5}\right]\right) \\
+ & (-1)^{\left|x_{3}\right|\left(\left|x_{1}\right|+\left|x_{2}\right|\right)}\left(d^{1} f\right)\left(x_{3},\left[x_{1}, x_{2}, x_{4}\right], x_{5}\right) \\
+ & \delta(-1)^{\left(\left|x_{1}\right|+\left|x_{2}\right|\right)\left(\left|x_{3}\right|+\left|x_{4}\right|\right)}\left(d^{1} f\right)\left(x_{3}, x_{4},\left[x_{1}, x_{2}, x_{5}\right]\right) .
\end{aligned}
$$

By Definition 23, we have

$$
\begin{aligned}
(55) & =(-1)^{\left(|f|+\left|x_{1}\right|+\left|x_{2}\right|+\left|x_{3}\right|\right)\left(\left|x_{4}\right|+\left|x_{5}\right|\right)} \theta\left(x_{4}, x_{5}\right)\left(d^{1} f\right) \\
\cdot & \left(x_{1}, x_{2}, x_{3}\right)=(-1)^{\left(|f|+\left|x_{1}\right|+\left|x_{2}\right|+\left|x_{3}\right|\right)\left(\left|x_{4}\right|+\left|x_{5}\right|\right)} \\
\cdot & \theta\left(x_{4}, x_{5}\right)\left\{-f\left(\left[x_{1}, x_{2}, x_{3}\right]+(-1)^{\left(|f|+\left|x_{1}\right|\right)\left(\left|x_{2}\right|+\left|x_{3}\right|\right)}\right.\right. \\
\cdot & \theta\left(x_{2}, x_{3}\right) f\left(x_{1}\right)+\delta(-1)^{|f|\left(\left|x_{1}\right|+\left|x_{2}\right|\right)} D\left(x_{1}, x_{2}\right) \\
\cdot & f\left(x_{3}\right)-\delta(-1)^{\left|x_{2}\right|\left|x_{3}\right|+|f|\left(\left|x_{1}\right|+\left|x_{3}\right|\right)} \theta\left(x_{1}, x_{3}\right) \\
\cdot & \left.f\left(x_{2}\right)\right\} \\
(56) & =-\delta(-1)^{\left(|f|+\left|x_{1}\right|+\left|x_{2}\right|\right)\left(\left|x_{3}\right|+\left|x_{5}\right|\right)+\left|x_{4}\right|\left|x_{5}\right|} \theta\left(x_{3}, x_{5}\right) \\
\cdot & \left(d^{1} f\right)\left(x_{1}, x_{2}, x_{4}\right) \\
= & -\delta(-1)^{\left(|f|+\left|x_{1}\right|+\left|x_{2}\right|\right)\left(\left|x_{3}\right|+\left|x_{5}\right|\right)+\left|x_{4}\right|\left|x_{5}\right|} \theta\left(x_{3}, x_{5}\right) \\
\cdot & \left\{-f\left(\left[x_{1}, x_{2}, x_{4}\right]\right)+(-1)^{\left(|f|+\left|x_{1}\right|\right)\left(\left|x_{2}\right|+\left|x_{4}\right|\right)} \theta\left(x_{2}, x_{4}\right)\right. \\
\cdot & f\left(x_{1}\right)+\delta(-1)^{|f|\left(\left|x_{1}\right|+\left|x_{2}\right|\right)} D\left(x_{1}, x_{2}\right) f\left(x_{4}\right) \\
- & \left.\delta(-1)^{\left|x_{2}\right|\left|x_{4}\right|+|f|\left(\left|x_{1}\right|+\left|x_{4}\right|\right)} \theta\left(x_{1}, x_{4}\right) f\left(x_{2}\right)\right\},
\end{aligned}
$$




$$
\begin{aligned}
& (57)=-\delta(-1)^{|f|\left(\left|x_{1}\right|+\left|x_{2}\right|\right)} D\left(x_{1}, x_{2}\right)\left(d^{1} f\right)\left(x_{3}, x_{4}, x_{5}\right) \\
& =-\delta(-1)^{|f|\left(\left|x_{1}\right|+\left|x_{2}\right|\right)} D\left(x_{1}, x_{2}\right)\left\{-f\left(\left[x_{3}, x_{4}, x_{5}\right]\right)\right. \\
& +(-1)^{\left(|f|+\left|x_{3}\right|\right)\left(\left|x_{4}\right|+\left|x_{5}\right|\right)} \theta\left(x_{4}, x_{5}\right) f\left(x_{3}\right) \\
& +\delta(-1)^{|f|\left(\left|x_{3}\right|+\left|x_{4}\right|\right)} D\left(x_{3}, x_{4}\right) f\left(x_{5}\right) \\
& \left.-\delta(-1)^{\left|x_{4}\right|\left|x_{5}\right|+|f|\left(\left|x_{3}\right|+\left|x_{5}\right|\right)} \theta\left(x_{3}, x_{5}\right) f\left(x_{4}\right)\right\}, \\
& (58)=(-1)^{\left(|f|+\left|x_{1}\right|+\left|x_{2}\right|\right)\left(\left|x_{3}\right|+\left|x_{4}\right|\right)} D\left(x_{3}, x_{4}\right)\left(d^{1} f\right) \\
& \cdot\left(x_{1}, x_{2}, x_{5}\right)=(-1)^{\left(|f|+\left|x_{1}\right|+\left|x_{2}\right|\right)\left(\left|x_{3}\right|+\left|x_{4}\right|\right)} D\left(x_{3}, x_{4}\right) \\
& \cdot\left\{-f\left(\left[x_{1}, x_{2}, x_{5}\right]\right)+(-1)^{\left(|f|+\left|x_{1}\right|\right)\left(\left|x_{2}\right|+\left|x_{5}\right|\right)} \theta\left(x_{2}, x_{5}\right)\right. \\
& \cdot f\left(x_{1}\right)+\delta(-1)^{|f|\left(\left|x_{1}\right|+\left|x_{2}\right|\right)} D\left(x_{1}, x_{2}\right) f\left(x_{5}\right) \\
& \left.-\delta(-1)^{\left|x_{2}\right|\left|x_{5}\right|+|f|\left(\left|x_{1}\right|+\left|x_{5}\right|\right)} \theta\left(x_{1}, x_{5}\right) f\left(x_{2}\right)\right\}, \\
& (59)=\left(d^{1} f\right)\left(\left[x_{1}, x_{2}, x_{3}\right], x_{4}, x_{5}\right)-\left(d^{1} f\right) \\
& \cdot\left(x_{1}, x_{2},\left[x_{3}, x_{4}, x_{5}\right]\right) \\
& =(-1)^{\left(|f|+\left|x_{1}\right|+\left|x_{2}\right|+\left|x_{3}\right|\right)\left(\left|x_{4}\right|+\left|x_{5}\right|\right)} \theta\left(x_{4}, x_{5}\right) \\
& \cdot f\left(\left[x_{1}, x_{2}, x_{3}\right]\right)-f\left(\left[\left[x_{1}, x_{2}, x_{3}\right], x_{4}, x_{5}\right]\right) \\
& +\delta(-1)^{|f|\left(\left|x_{1}\right|+\left|x_{2}\right|+\left|x_{3}\right|+\left|x_{4}\right|\right)} D\left(\left[x_{1}, x_{2}, x_{3}\right], x_{4}\right) \\
& \cdot f\left(x_{5}\right)-\delta(-1)^{\left|x_{4}\right|\left|x_{5}\right|+|f|\left(\left|x_{1}\right|+\left|x_{2}\right|+\left|x_{3}\right|+\left|x_{5}\right|\right)} \\
& \cdot \theta\left(\left[x_{1}, x_{2}, x_{3}\right], x_{5}\right) f\left(x_{4}\right) \\
& -(-1)^{\left(|f|+\left|x_{1}\right|\right)\left(\left|x_{2}\right|+\left|x_{3}\right|+\left|x_{4}\right|+\left|x_{5}\right|\right)} \theta\left(x_{2},\left[x_{3}, x_{4}, x_{5}\right]\right) \\
& \cdot f\left(x_{1}\right)+f\left(\left[x_{1}, x_{2},\left[x_{3}, x_{4}, x_{5}\right]\right]\right) \\
& -\delta(-1)^{|f|\left(\left|x_{1}\right|+\left|x_{2}\right|\right)} D\left(x_{1}, x_{2}\right) f\left(\left[x_{3}, x_{4}, x_{5}\right]\right) \\
& +\delta(-1)^{|f|\left(\left|x_{1}\right|+\left|x_{3}\right|+\left|x_{4}\right|+\left|x_{5}\right|\right)+\left|x_{2}\right|\left(\left|x_{3}\right|+\left|x_{4}\right|+\left|x_{5}\right|\right)} \\
& \cdot \theta\left(x_{1},\left[x_{3}, x_{4}, x_{5}\right],\right) f\left(x_{2}\right), \\
& (60)=(-1)^{\left|x_{3}\right|\left(\left|x_{1}\right|+\left|x_{2}\right|\right)}\left(d^{1} f\right)\left(x_{3},\left[x_{1}, x_{2}, x_{4}\right], x_{5}\right) \\
& =(-1)^{\left|x_{3}\right|\left(\left|x_{1}\right|+\left|x_{2}\right|\right)+\left(|f|+\left|x_{3}\right|\right)\left(\left|x_{1}\right|+\left|x_{2}\right|+\left|x_{4}\right|+\left|x_{5}\right|\right)} \\
& \cdot \theta\left(\left[x_{1}, x_{2}, x_{4}\right], x_{5}\right) f\left(x_{3}\right)-(-1)^{\left|x_{3}\right|\left(\left|x_{1}\right|+\left|x_{2}\right|\right)} \\
& \cdot f\left(\left[x_{3},\left[x_{1}, x_{2}, x_{4}\right], x_{5}\right]\right) \\
& +\delta(-1)^{\left|x_{3}\right|\left(\left|x_{1}\right|+\left|x_{2}\right|\right)+|f|\left(\left|x_{1}\right|+\left|x_{2}\right|+\left|x_{3}\right|+\left|x_{4}\right|\right.} \\
& \cdot D\left(x_{3},\left[x_{1}, x_{2}, x_{4}\right]\right) f\left(x_{5}\right) \\
& -\delta(-1)^{\left(|f|+\left|x_{1}\right|+\left|x_{2}\right|\right)\left(\left|x_{3}\right|+\left|x_{5}\right|\right)+\left|x_{4}\right|\left|x_{5}\right|} \theta\left(x_{3}, x_{5}\right) \\
& \cdot f\left(\left[x_{1}, x_{2}, x_{4}\right]\right) \text {, }
\end{aligned}
$$

$$
\begin{aligned}
\Lambda_{1}= & (-1)^{\left(\left|x_{2}\right|+\left|x_{3}\right|\right)\left(\left|x_{4}\right|+\left|x_{5}\right|\right)} \theta\left(x_{4}, x_{5}\right) \theta\left(x_{2}, x_{3}\right) \\
& -\delta(-1)^{\left|x_{2}\right|\left|x_{3}\right|+\left(\left|x_{2}\right|+\left|x_{4}\right|\right)\left|x_{5}\right|} \theta\left(x_{3}, x_{5}\right) \theta\left(x_{2}, x_{4}\right) \\
& -\theta\left(x_{2},\left[x_{3}, x_{4}, x_{5}\right]\right) \\
& +\delta(-1)^{\left|x_{2}\right|\left(\left|x_{3}\right|+\left|x_{4}\right|\right)} D\left(x_{3}, x_{4}\right) \theta\left(x_{2}, x_{5}\right)=0,
\end{aligned}
$$




$$
\begin{aligned}
\Lambda_{2}= & (-1)^{\left(\left|x_{1}\right|+\left|x_{3}\right|\right)\left(\left|x_{4}\right|+\left|x_{5}\right|\right)} \theta\left(x_{4}, x_{5}\right) \theta\left(x_{1}, x_{3}\right) \\
& -\delta(-1)^{\left|x_{1}\right|\left|x_{3}\right|+\left|x_{5}\right|\left(\left|x_{1}\right|+\left|x_{4}\right|\right)} \theta\left(x_{3}, x_{5}\right) \theta\left(x_{1}, x_{4}\right) \\
& -\theta\left(x_{1},\left[x_{3}, x_{4}, x_{5}\right]\right) \\
& +\delta(-1)^{\left|x_{1}\right|\left(\left|x_{3}\right|+\left|x_{4}\right|\right)} D\left(x_{3}, x_{4}\right) \theta\left(x_{1}, x_{5}\right)=0,
\end{aligned}
$$

$$
\begin{aligned}
\Lambda_{3}= & (-1)^{\left(\left|x_{1}\right|+\left|x_{2}\right|\right)\left(\left|x_{4}\right|+\left|x_{5}\right|\right)} \theta\left(x_{4}, x_{5}\right) D\left(x_{1}, x_{2}\right) \\
& -\delta D\left(x_{1}, x_{2}\right) \theta\left(x_{4}, x_{5}\right)+\theta\left(\left[x_{1}, x_{2}, x_{4}\right], x_{5}\right) \\
& +\delta(-1)^{\left|x_{4}\right|\left(\left|x_{1}\right|+\left|x_{2}\right|\right)} \theta\left(x_{4},\left[x_{1}, x_{2}, x_{5}\right]\right)=0,
\end{aligned}
$$

by Eq. (34)

$$
\begin{aligned}
\Lambda_{4}= & (-1)^{\left(\left|x_{1}\right|+\left|x_{2}\right|\right)\left(\left|x_{3}\right|+\left|x_{5}\right|\right)} \theta\left(x_{3}, x_{5}\right) D\left(x_{1}, x_{2}\right) \\
& -\delta D\left(x_{1}, x_{2}\right) \theta\left(x_{3}, x_{5}\right)+\theta\left(\left[x_{1}, x_{2}, x_{3}\right], x_{5}\right) \\
& +\delta(-1)^{\left|x_{3}\right|\left(\left|x_{1}\right|+\left|x_{2}\right|\right)} \theta\left(x_{3},\left[x_{1}, x_{2}, x_{5}\right]\right)=0,
\end{aligned}
$$

$$
\begin{aligned}
\Lambda_{5}= & D\left(\left[x_{1}, x_{2}, x_{3}\right], x_{4}\right) \\
& +(-1)^{\left|x_{3}\right|\left(\left|x_{1}\right|+\left|x_{2}\right|\right)} D\left(x_{3},\left[x_{1}, x_{2}, x_{4}\right]\right) \\
& -\delta D\left(x_{1}, x_{2}\right) D\left(x_{3}, x_{4}\right) \\
& +(-1)^{\left(\left|x_{1}\right|+\left|x_{2}\right|\right)\left(\left|x_{3}\right|+\left|x_{4}\right|\right)} D\left(x_{3}, x_{4}\right) D\left(x_{1}, x_{2}\right)
\end{aligned}
$$

$=0$. by Eq. (35)

It follows that $d^{3}\left(d^{1} f\right)\left(x_{1}, x_{2}, x_{3}, x_{4}, x_{5}\right)=0$, as desired. And this finishes the proof.

For $n=1,2$, the map $f \in C_{\delta}^{n}(T, V)$ is called an $n$-cocycle if $d^{n} f=0$. We denote by $Z_{\delta}^{n}(T, V)$ the subspace spanned by $n$-cocycles and $B_{\delta}^{n}(T, V)=d^{n-2} C_{\delta}^{n-2}(T, V)$. By Theorem 24, $B_{\delta}^{n}(T, V)$ is a subspace of $Z_{\delta}^{n}(T, V)$. Therefore, we can define a cohomology space $H_{\delta}^{n}(T, V)$ of the $\delta$-Jordn Lie supertriple system $T$ as the factor space $Z_{\delta}^{n}(T, V) / B_{\delta}^{n}(T, V)$.

\section{1-Parameter Formal Deformations of $\delta$-Jordan Lie Supertriple Systems}

Let $T$ be a $\delta$-Jordan Lie supertriple system and $k[[t]]$ the power series ring in one variable $t$ with coefficients in $k$. Assume that $T[[t]]$ is the set of formal series whose coefficients are elements of the vector space $T$.

Definition 25. Let $T$ be a $\delta$-Jordan Lie supertriple system. A 1-parameter formal deformation of $T$ is a formal power series $f_{t}: T[[t]] \times T[[t]] \times T[[t]] \longrightarrow T[[t]]$ given by

$$
\begin{aligned}
f_{t}= & \sum_{i \geq 0} f_{i}\left(x_{1}, x_{2}, x_{3}\right) t^{i} \\
= & f_{0}\left(x_{1}, x_{2}, x_{3}\right)+f_{1}\left(x_{1}, x_{2}, x_{3}\right) t \\
& +f_{2}\left(x_{1}, x_{2}, x_{3}\right) t^{2}+\cdots,
\end{aligned}
$$

where each $f_{i}$ is a $k$-trilinear map $f_{i}: T \times T \times T \longrightarrow$ $T$ (extended to be $k[[t]]$-trilinear) and $f_{0}\left(x_{1}, x_{2}, x_{3}\right)=$ $\left[x_{1}, x_{2}, x_{3}\right]$, satisfying the following axioms:

$$
\begin{aligned}
& \left|f_{t}\left(x_{1}, x_{2}, x_{3}\right)\right|=\left|x_{1}\right|+\left|x_{2}\right|+\left|x_{3}\right| \\
& f_{t}\left(x_{2}, x_{1}, x_{3}\right)=-\delta(-1)^{\left|x_{1}\right|\left|x_{2}\right|} f_{t}\left(x_{1}, x_{2}, x_{3}\right) ; \\
& (-1)^{\left|x_{1}\right|\left|x_{3}\right|} f_{t}\left(x_{1}, x_{2}, x_{3}\right)+(-1)^{\left|x_{2}\right|\left|x_{1}\right|} f_{t}\left(x_{2}, x_{3}, x_{1}\right) \\
& \quad+(-1)^{\left|x_{3}\right|\left|x_{2}\right|} f_{t}\left(x_{3}, x_{1}, x_{2}\right)=0 ; \\
& f_{t}\left(x_{1}, x_{2}, f_{t}\left(x_{3}, x_{4}, x_{5}\right)\right)=(-1)^{\left|x_{3}\right|\left(\left|x_{1}\right|+\left|x_{2}\right|\right)} \\
& \quad \cdot f_{t}\left(x_{3}, f_{t}\left(x_{1}, x_{2}, x_{4}\right), x_{5}\right) \\
& \quad+f_{t}\left(f_{t}\left(x_{1}, x_{2}, x_{3}\right), x_{4}, x_{5}\right) \\
& \quad+\delta(-1)^{\left(\left|x_{1}\right|+\left|x_{2}\right|\right)\left(\left|x_{3}\right|+\left|x_{4}\right|\right)} f_{t}\left(x_{3}, x_{4}, f_{t}\left(x_{1}, x_{2}, x_{5}\right)\right) .
\end{aligned}
$$

Remark 26. Equations (72)-(75) are equivalent to $(n=$ $0,1,2, \cdots)$

$$
\begin{aligned}
& \left|f_{i}\left(x_{2}, x_{1}, x_{3}\right)\right|=\left|x_{1}\right|+\left|x_{2}\right|+\left|x_{3}\right| \\
& f_{i}\left(x_{2}, x_{1}, x_{3}\right)=-\delta(-1)^{\left|x_{1}\right|\left|x_{2}\right|} f_{i}\left(x_{1}, x_{2}, x_{3}\right) \\
& (-1)^{\left|x_{1}\right|\left|x_{3}\right|} f_{i}\left(x_{1}, x_{2}, x_{3}\right)+(-1)^{\left|x_{2}\right|\left|x_{1}\right|} f_{i}\left(x_{2}, x_{3}, x_{1}\right) \\
& \quad+(-1)^{\left|x_{3}\right|\left|x_{2}\right|} f_{i}\left(x_{3}, x_{1}, x_{2}\right)=0 ; \\
& \sum_{i+j=n} f_{i}\left(x_{1}, x_{2}, f_{j}\left(x_{3}, x_{4}, x_{5}\right)\right)=\sum_{i+j=n}\left((-1)^{\left|x_{3}\right|\left(\left|x_{1}\right|+\left|x_{2}\right|\right)}\right. \\
& \quad \cdot f_{i}\left(x_{3}, f_{j}\left(x_{1}, x_{2}, x_{4}\right), x_{5}\right) \\
& \quad+f_{i}\left(f_{j}\left(x_{1}, x_{2}, x_{3}\right), x_{4}, x_{5}\right) \\
& \quad+\delta(-1)^{\left(\left|x_{1}\right|+\left|x_{2}\right|\right)\left(\left|x_{3}\right|+\left|x_{4}\right|\right)} \\
& \left.\quad \cdot f_{i}\left(x_{3}, x_{4}, f_{j}\left(x_{1}, x_{2}, x_{5}\right)\right)\right) .
\end{aligned}
$$

Furthermore, we can rewrite the deformation Equation (79) by the equality $\sum_{i+j=n} f_{i} f_{j}=0$, where

$$
\begin{aligned}
& f_{i} f_{j}\left(x_{1}, x_{2}, x_{3}, x_{4}, x_{5}\right)=-f_{i}\left(x_{1}, x_{2}, f_{j}\left(x_{3}, x_{4}, x_{5}\right)\right) \\
& \quad+(-1)^{\left|x_{3}\right|\left(\left|x_{1}\right|+\left|x_{2}\right|\right)} f_{i}\left(x_{3}, f_{j}\left(x_{1}, x_{2}, x_{4}\right), x_{5}\right) \\
& \quad+f_{i}\left(f_{j}\left(x_{1}, x_{2}, x_{3}\right), x_{4}, x_{5}\right) \\
& \quad+\delta(-1)^{\left(\left|x_{1}\right|+\left|x_{2}\right|\right)\left(\left|x_{3}\right|+\left|x_{4}\right|\right)} f_{i}\left(x_{3}, x_{4}, f_{j}\left(x_{1}, x_{2}, x_{5}\right)\right) .
\end{aligned}
$$

When $n=1$, (79) is equivalent to $f_{0} f_{1}+f_{1} f_{0}=0$. When $n \geq 2$, (79) is equivalent to $-\left(f_{0} f_{n}+f_{n} f_{0}\right)=f_{1} f_{n-1}+f_{2} f_{n-2}+$ $\cdots+f_{n-1} f_{1}$.

By Example 19, $(T, \theta)$ is the adjoint representation of $T$ itself, where $\theta(a, b)(x)=(-1)^{|x|(|a|+|b|)}[x, a, b]$ and $D(a, b)(x)=\delta[a, b, x]$. It is easy to see that $f_{i} \in C_{\delta}^{3}(T, V)$ and 
therefore $f_{i} f_{j} \in C_{\delta}^{5}(T, V)$. Since $f_{0}\left(x_{1}, x_{2}, x_{3}\right)=\left[x_{1}, x_{2}, x_{3}\right]$, we have

$$
\begin{aligned}
f_{0} & f_{1}\left(x_{1}, x_{2}, x_{3}, x_{4}, x_{5}\right)=-f_{0}\left(x_{1}, x_{2}, f_{1}\left(x_{3}, x_{4}, x_{5}\right)\right) \\
& +(-1)^{\left|x_{3}\right|\left(\left|x_{1}\right|+\left|x_{2}\right|\right)} f_{0}\left(x_{3}, f_{1}\left(x_{1}, x_{2}, x_{4}\right), x_{5}\right) \\
& +f_{0}\left(f_{1}\left(x_{1}, x_{2}, x_{3}\right), x_{4}, x_{5}\right) \\
& +\delta(-1)^{\left(\left|x_{1}\right|+\left|x_{2}\right|\right)\left(\left|x_{3}\right|+\left|x_{4}\right|\right)} f_{0}\left(x_{3}, x_{4}, f_{1}\left(x_{1}, x_{2}, x_{5}\right)\right) \\
& =-\left[x_{1}, x_{2}, f_{1}\left(x_{3}, x_{4}, x_{5}\right)\right]+(-1)^{\left|x_{3}\right|\left(\left|x_{1}\right|+\left|x_{2}\right|\right)} \\
& \cdot\left[x_{3}, f_{1}\left(x_{1}, x_{2}, x_{4}\right), x_{5}\right]+\left[f_{1}\left(x_{1}, x_{2}, x_{3}\right), x_{4}, x_{5}\right] \\
& +\delta(-1)^{\left(\left|x_{1}\right|+\left|x_{2}\right|\right)\left(\left|x_{3}\right|+\left|x_{4}\right|\right)}\left[x_{3}, x_{4}, f_{1}\left(x_{1}, x_{2}, x_{5}\right)\right] \\
& =-\delta D\left(x_{1}, x_{2}\right) f\left(x_{3}, x_{4}, x_{5}\right) \\
& -\delta(-1)^{\left(\left|x_{1}\right|+\left|x_{2}\right|\right)\left(\left|x_{3}\right|+\left|x_{5}\right|\right)+\left|x_{4}\right|\left|x_{5}\right|} D\left(x_{3}, x_{4}\right) \\
\cdot & f\left(x_{1}, x_{2}, x_{5}\right)+(-1)^{\left(\left|x_{1}\right|+\left|x_{2}\right|+\left|x_{3}\right|\right)\left(\left|x_{4}\right|+\left|x_{5}\right|\right)} \\
\cdot & \theta\left(x_{4}, x_{5}\right) f\left(x_{1}, x_{2}, x_{3}\right)+(-1)^{\left(\left|x_{1}\right|+\left|x_{2}\right|\right)\left(\left|x_{3}\right|+\left|x_{4}\right|\right)} \\
& \cdot D\left(x_{3}, x_{4}\right) f\left(x_{1}, x_{2}, x_{5}\right)
\end{aligned}
$$

Similarly, we have

$$
\begin{aligned}
f_{1} & f_{0}\left(x_{1}, x_{2}, x_{3}, x_{4}, x_{5}\right) \\
= & -f_{1}\left(x_{1}, x_{2}, f_{0}\left(x_{3}, x_{4}, x_{5}\right)\right) \\
& +(-1)^{\left|x_{3}\right|\left(\left|x_{1}\right|+\left|x_{2}\right|\right)} f_{1}\left(x_{3}, f_{0}\left(x_{1}, x_{2}, x_{4}\right), x_{5}\right) \\
& +f_{1}\left(f_{0}\left(x_{1}, x_{2}, x_{3}\right), x_{4}, x_{5}\right) \\
& +\delta(-1)^{\left(\left|x_{1}\right|+\left|x_{2}\right|\right)\left(\left|x_{3}\right|+\left|x_{4}\right|\right)} f_{1}\left(x_{3}, x_{4}, f_{0}\left(x_{1}, x_{2}, x_{5}\right)\right) \\
= & -f_{1}\left(x_{1}, x_{2},\left[x_{3}, x_{4}, x_{5}\right]\right) \\
& +(-1)^{\left|x_{3}\right|\left(\left|x_{1}\right|+\left|x_{2}\right|\right)} f_{1}\left(x_{3},\left[x_{1}, x_{2}, x_{4}\right], x_{5}\right) \\
& +f_{1}\left(\left[x_{1}, x_{2}, x_{3}\right], x_{4}, x_{5}\right) \\
& +\delta(-1)^{\left(\left|x_{1}\right|+\left|x_{2}\right|\right)\left(\left|x_{3}\right|+\left|x_{4}\right|\right)} f_{1}\left(x_{3}, x_{4},\left[x_{1}, x_{2}, x_{5}\right]\right) .
\end{aligned}
$$

It follows that

$$
\begin{gathered}
\left(f_{0} f_{1}+f_{1} f_{0}\right)\left(x_{1}, x_{2}, x_{3}, x_{4}, x_{5}\right) \\
=d^{3} f_{1}\left(x_{1}, x_{2}, x_{3}, x_{4}, x_{5}\right) .
\end{gathered}
$$

Therefore, we deduce $d^{3} f_{1}=0$ since $f_{0} f_{1}+f_{1} f_{0}=0$. Also we can obtain $-d^{3} f_{n}=f_{1} f_{n-1}+f_{2} f_{n-2}+\cdots+f_{n-1} f_{1}$. And $f_{1}$ is called the infinitesimal deformation of $f_{t}$.

Definition 27. Let $T$ be a $\delta$-Jordan Lie supertriple system. Two 1-parameter formal deformations $f_{t}$ and $f_{t}^{\prime}$ of $T$ are said to be equivalent, denoted by $f_{t} \sim f_{t}^{\prime}$, if there exists a formal isomorphism of $k[[t]]$-modules

$$
\begin{aligned}
\phi_{t}(x) & =\sum_{i \geq 0} \phi_{i}(x) t^{i}:\left(T[[t]], f_{t}, \delta\right) \\
& \longrightarrow\left(T[[t]], f_{t}^{\prime}, \delta\right)
\end{aligned}
$$

where $\phi_{0}=i d_{T}, \phi_{i}: T \longrightarrow T$ is an $k$-linear map (extended to be $k[[t]]-$ linear) such that

$$
\begin{array}{r}
\phi_{t} f_{t}\left(x_{1}, x_{2}, x_{3}\right)=f_{t}^{\prime}\left(\phi_{t}\left(x_{1}\right), \phi_{t}\left(x_{2}\right), \phi_{t}\left(x_{3}\right)\right), \\
\forall x_{1}, x_{2}, x_{3} \in T .
\end{array}
$$

In particular, if $f_{1}=f_{2}=\cdots=0$, then $f_{1}=f_{0}$ is called the null deformation. If $f_{t} \sim f_{0}$, then $f_{t}$ is called the trivial deformation. If every 1-parameter formal deformation $f_{t}$ is trivial, then $T$ is called an analytically rigid $\delta$-Jordan Lie supertriple system.

Theorem 28. Let $f_{t}=\sum_{i \geq 0} f_{i}\left(x_{1}, x_{2}, x_{3}\right) t^{i}$ and $f_{t}^{\prime}=$ $\sum_{i \geq 0} f_{i}^{\prime}\left(x_{1}, x_{2}, x_{3}\right) t^{i}$ be two equivalent 1-parameter formal deformations of $T$. Then the infinitesimal deformations $f_{1}$ and $f_{1}^{\prime}$ belong to the same cohomology class in $H_{\delta}^{3}(T, T)$.

Proof. By the assumption that $f_{1}$ and $f_{1}^{\prime}$ are equivalent, there exists a formal isomorphism $\phi_{t}(x)=\sum_{i \geq 0} \phi_{i}(x) t^{i}$ of $k[[t]]$ modules satisfying

$$
\begin{gathered}
\sum_{i \geq 0} \phi_{i}\left(\sum_{j \geq 0} f_{j}\left(x_{1}, x_{2}, x_{3}\right) t^{j}\right)=\sum_{i \geq 0} f_{i}^{\prime}\left(\sum_{k \geq 0} \phi_{k}\left(x_{1}\right) t^{k},\right. \\
\left.\sum_{l \geq 0} \phi_{l}\left(x_{2}\right) t^{l}, \sum_{m \geq 0} \phi_{m}\left(x_{3}\right) t^{m}\right) t^{i}
\end{gathered}
$$

for any $x_{1}, x_{2}, x_{3} \in T$. Compared with the coefficients of $t^{1}$ for two sides of the above equation, we have

$$
\begin{aligned}
& f_{1}\left(x_{1}, x_{2}, x_{3}\right)+\phi_{1}\left(\left[x_{1}, x_{2}, x_{3}\right]\right) \\
& =f_{1}^{\prime}\left(x_{1}, x_{2}, x_{3}\right)+\left[\phi_{1}\left(x_{1}\right), x_{2}, x_{3}\right] \\
& \quad+\left[x_{1}, \phi_{1}\left(x_{2}\right), x_{3}\right]+\left[x_{1}, x_{2}, \phi_{1}\left(x_{3}\right)\right] .
\end{aligned}
$$

It follows that

$$
\begin{aligned}
f_{1}\left(x_{1}, x_{2}, x_{3}\right)-f_{1}^{\prime}\left(x_{1}, x_{2}, x_{3}\right) \\
=\left[\phi_{1}\left(x_{1}\right), x_{2}, x_{3}\right]+\left[x_{1}, \phi_{1}\left(x_{2}\right), x_{3}\right] \\
\quad+\left[x_{1}, x_{2}, \phi_{1}\left(x_{3}\right)\right]-\phi_{1}\left(\left[x_{1}, x_{2}, x_{3}\right]\right) \\
=(-1)^{\left|x_{1}\right|\left(\left|x_{2}\right|+\left|x_{3}\right|\right)} \theta\left(x_{2}, x_{3}\right) \phi_{1}\left(x_{1}\right) \\
\quad-\phi_{1}\left(\left[x_{1}, x_{2}, x_{3}\right]\right)+\delta D\left(x_{1}, x_{2}\right) \phi_{1}\left(x_{3}\right) \\
\quad-\delta(-1)^{\left|x_{2}\right|\left|x_{3}\right|} \theta\left(x_{1}, x_{3}\right) \phi_{1}\left(x_{2}\right) \\
=d^{1} \phi_{1}\left(x_{1}, x_{2}, x_{3}\right) .
\end{aligned}
$$


So $f_{1}-f_{1}^{\prime}=d^{1} \phi_{1} \in B_{\delta}^{3}(T, T)$, as desired. The proof is completed.

Theorem 29. Let $T$ be a $\delta$-Jordan Lie supertriple system with $H_{\delta}^{3}(T, T)=0$; then $T$ is analytically rigid.

Proof. Let $f_{t}=\sum_{i \geq 0} f_{i} t^{i}$ be a 1-parameter formal deformation of $T$. Then $d^{3} f_{n}=f_{1} f_{n-1}+f_{2} f_{n-2}+\cdots+f_{n-1} f_{1}=0$. By the assumption $H_{\delta}^{3}(T, T)=0$, we have $f_{n} \in Z_{\delta}^{3}(T, T)=B_{\delta}^{3}(T, T)$; that is, there exits $g_{n} \in C_{\delta}^{1}(T, T)$ such that $f_{n}=d^{1} g^{n}$.

$$
\begin{aligned}
& \text { Set } \phi_{t}=i d_{T}-g_{n} t^{n} ; \text { then } \\
& \begin{aligned}
\phi_{t} & \left(i d_{T}+g_{n} t^{n}+g_{n}^{2} t^{2 n}+g_{n}^{3} t^{3 n}+\cdots\right) \\
= & \left(i d_{T}-g_{n} t^{n}\right)\left(i d_{T}+g_{n} t^{n}+g_{n}^{2} t^{2 n}+g_{n}^{3} t^{3 n}+\cdots\right) \\
= & \left(i d_{T}+g_{n} t^{n}+g_{n}^{2} t^{2 n}+g_{n}^{3} t^{3 n}+\cdots\right) \\
& -\left(g_{n} t^{n}+g_{n}^{2} t^{2 n}+g_{n}^{3} t^{3 n}+\cdots\right)=i d_{T} .
\end{aligned}
\end{aligned}
$$

Similarly, one may check that $\left(i d_{T}+g_{n} t^{n}+g_{n}^{2} t^{2 n}+g_{n}^{3} t^{3 n}+\right.$ $\cdots) \phi_{t}=i d_{T}$. So $\phi_{t}:\left(T[[t]], f_{t}, \delta\right) \longrightarrow\left(T[[t]], f_{t}^{\prime}, \delta\right)$ is a linear isomorphism. Thus we can define another 1-parameter formal deformation by $\phi_{t}^{-1}$ in the form of

$$
f_{t}^{\prime}\left(x_{1}, x_{2}, x_{3}\right)=\phi_{t}^{-1} f_{i}\left(\phi_{t}\left(x_{1}\right), \phi_{t}\left(x_{2}\right), \phi_{t}\left(x_{3}\right)\right) .
$$

Obviously, $f_{t} \sim f_{t}^{\prime}$. Set $f_{t}^{\prime}=\sum_{i \geq 0} f_{i}^{\prime} t^{i}$; then we have

$$
\begin{aligned}
\sum_{i \geq 0} & f_{i}^{\prime}\left(x_{1}, x_{2}, x_{3}\right) t^{i}-\sum_{i \geq 0} g_{n} f_{i}\left(x_{1}, x_{2}, x_{3}\right) t^{i+n}=f_{0}\left(x_{1},\right. \\
& \left.x_{2}, x_{3}\right)-\left\{f_{0}\left(g_{n}\left(x_{1}\right), x_{2}, x_{3}\right)+f_{0}\left(x_{1}, g_{n}\left(x_{2}\right), x_{3}\right)\right. \\
& \left.+f_{0}\left(x_{1}, x_{2}, g_{n}\left(x_{3}\right)\right)\right\} t^{n} \\
& +\left\{f_{0}\left(g_{n}\left(x_{1}\right), g_{n}\left(x_{2}\right), x_{3}\right)\right. \\
& +f_{0}\left(x_{1}, g_{n}\left(x_{2}\right), g_{n}\left(x_{3}\right)\right) \\
& \left.+f_{0}\left(x_{1}, g_{n}\left(x_{2}\right), g_{n}\left(x_{3}\right)\right)\right\} t^{2 n}+f_{0}\left(g_{n}\left(x_{1}\right),\right. \\
& \left.g_{n}\left(x_{2}\right), g_{n}\left(x_{3}\right)\right) t^{3 n}+\sum_{i \geq n} f_{i}\left(x_{1}, x_{2}, x_{3}\right) t^{i} \\
& -\sum_{i \geq n}\left\{f_{i}\left(g_{n}\left(x_{1}\right), x_{2}, x_{3}\right)+f_{i}\left(x_{1}, g_{n}\left(x_{2}\right), x_{3}\right)\right. \\
& \left.+f_{i}\left(x_{1}, x_{2}, g_{n}\left(x_{3}\right)\right)\right\} t^{i+n} \\
& +\sum_{i \geq n}\left\{f_{i}\left(g_{n}\left(x_{1}\right), g_{n}\left(x_{2}\right), x_{3}\right)\right. \\
& +f_{i}\left(x_{1}, g_{n}\left(x_{2}\right), g_{n}\left(x_{3}\right)\right) \\
& \left.+f_{i}\left(g_{n}\left(x_{1}\right), x_{2}, g_{n}\left(x_{3}\right)\right)\right\} t^{i+2 n} \\
& -\sum_{i \geq n} f_{i}\left(g_{n}\left(x_{1}\right), g_{n}\left(x_{2}\right), g_{n}\left(x_{3}\right)\right) t^{i+3 n} .
\end{aligned}
$$

By the above equation, it follows that

$$
\begin{aligned}
f_{0}^{\prime}\left(x_{1}, x_{2}, x_{3}\right)=f_{0}\left(x_{1}, x_{2}, x_{3}\right)=\left[x_{1}, x_{2}, x_{3}\right], \\
f_{1}^{\prime}\left(x_{1}, x_{2}, x_{3}\right)=f_{1}^{\prime}\left(x_{1}, x_{2}, x_{3}\right)=\cdots \\
=f_{n-1}^{\prime}\left(x_{1}, x_{2}, x_{3}\right)=0, \\
f_{n}^{\prime}\left(x_{1}, x_{2}, x_{3}\right)-g_{n}\left(\left[x_{1}, x_{2}, x_{3}\right]\right) \\
=f_{n}\left(x_{1}, x_{2}, x_{3}\right)-\left[g_{n}\left(x_{1}\right), x_{2}, x_{3}\right] \\
\quad-\left[x_{1}, g_{n}\left(x_{2}\right), x_{3}\right]-\left[x_{1}, x_{2}, g_{n}\left(x_{3}\right)\right] \\
=f_{n}\left(x_{1}, x_{2}, x_{3}\right) \\
\quad-(-1)^{\left|x_{1}\right|\left(\left|x_{2}\right|+\left|x_{3}\right|\right)} \theta\left(x_{2}, x_{3}\right) g_{n}\left(x_{1}\right) \\
\quad+\delta(-1)^{\left|x_{2}\right|\left|x_{3}\right|} \theta\left(x_{1}, x_{3}\right) g_{n}\left(x_{2}\right) \\
\quad-\delta D\left(x_{1}, x_{2}\right) g_{n}\left(x_{3}\right) .
\end{aligned}
$$

Therefore, we deduce

$$
\begin{aligned}
f_{n}^{\prime}\left(x_{1}, x_{2}, x_{3}\right) & =f_{n}\left(x_{1}, x_{2}, x_{3}\right)-d^{1} g_{n}\left(x_{1}, x_{2}, x_{3}\right) \\
& =0 .
\end{aligned}
$$

It follows that $f_{t}^{\prime}=f_{0}+\sum_{i \geq n+1} f_{i}^{\prime} t^{i}$. By induction, we have $f_{t} \sim$ $f_{0}$; that is, $T$ is analytically rigid. The proof is finished.

\section{Nijenhuis Operators of $\delta$-Jordan Lie Supertriple Systems}

In this section, we introduce the notion of Nijenhuis operators for $\delta$-Jordan Lie supertriple systems. Also, we give trivial deformations of this kind of operators.

Let $T$ be a $\delta$-Jordan Lie supertriple system and let $\psi$ : $T \times T \times T \longrightarrow T$ be an even trilinear map. Consider a $\lambda$ parametrized family of linear operations:

$$
\left[x_{1}, x_{2}, x_{3}\right]_{\lambda}=\left[x_{1}, x_{2}, x_{3}\right]+\lambda \psi\left(x_{1}, x_{2}, x_{3}\right),
$$

for any $x_{1}, x_{2}, x_{3} \in T$, where $\lambda$ is a formal variable.

If $[\cdot, \cdot, \cdot]_{\lambda}$ endow $T$ with a $\delta$-Jordan Lie supertriple system structure which is denoted by $T_{\lambda}$, then we call that $\psi$ generates a $\lambda$-parameter infinitesimal deformation of the $\delta$-Jordan Lie supertriple system $T$.

Theorem 30. Let $T$ be a $\delta$-Jordan Lie supertriple system. Then $T_{\lambda}$ is a $\delta$-Jordan Lie supertriple system if and only if

(i) $\psi$ itself defines a $\delta$-Jordan Lie supertriple system structure on $T$;

(ii) $\psi$ is a 3-cocycle of $T$.

Proof. Assume that $T_{\lambda}$ is a $\delta$-Jordan Lie supertriple system. For any $x_{1}, x_{2}, x_{3} \in T$, we have 


$$
\begin{aligned}
{\left[x_{2}, x_{1}, x_{3}\right]_{\lambda}=} & {\left[x_{2}, x_{1}, x_{3}\right]+\lambda \psi\left(x_{2}, x_{1}, x_{3}\right) } \\
= & -\delta(-1)^{\left|x_{1}\right|\left|x_{2}\right|}\left[x_{1}, x_{2}, x_{3}\right] \\
& +\lambda \psi\left(x_{2}, x_{1}, x_{3}\right), \\
{\left[x_{2}, x_{1}, x_{3}\right]_{\lambda}=} & -\delta(-1)^{\left|x_{1}\right|\left|x_{2}\right|}\left[x_{1}, x_{2}, x_{3}\right]_{\lambda} \\
= & -\delta(-1)^{\left|x_{1}\right|\left|x_{2}\right|}\left[x_{1}, x_{2}, x_{3}\right] \\
& -\delta(-1)^{\left|x_{1}\right|\left|x_{2}\right|} \lambda \psi\left(x_{1}, x_{2}, x_{3}\right) .
\end{aligned}
$$

It follows that

$$
\psi\left(x_{2}, x_{1}, x_{3}\right)=-\delta(-1)^{\left|x_{1}\right|\left|x_{2}\right|} \psi\left(x_{1}, x_{2}, x_{3}\right) .
$$

For (3), we have

$$
\begin{aligned}
\lambda & \left((-1)^{\left|x_{1}\right|\left|x_{3}\right|} \psi\left(x_{1}, x_{2}, x_{3}\right)+(-1)^{\left|x_{2}\right|\left|x_{1}\right|} \psi\left(x_{2}, x_{3}, x_{1}\right)\right. \\
& \left.+(-1)^{\left|x_{3}\right|\left|x_{2}\right|} \psi\left(x_{3}, x_{1}, x_{2}\right)\right)=(-1)^{\left|x_{1}\right|\left|x_{3}\right|} \\
& \cdot\left[x_{1}, x_{2}, x_{3}\right]_{\lambda}+(-1)^{\left|x_{2}\right|\left|x_{1}\right|}\left[x_{2}, x_{3}, x_{1}\right]_{\lambda} \\
& +(-1)^{\left|x_{3}\right|\left|x_{2}\right|}\left[x_{3}, x_{1}, x_{2}\right]_{\lambda}-(-1)^{\left|x_{1}\right|\left|x_{3}\right|}\left[x_{1}, x_{2}, x_{3}\right] \\
& -(-1)^{\left|x_{2}\right|\left|x_{1}\right|}\left[x_{2}, x_{3}, x_{1}\right]-(-1)^{\left|x_{3}\right|\left|x_{2}\right|}\left[x_{3}, x_{1}, x_{2}\right] \\
& =0
\end{aligned}
$$

as desired. The last equality holds since $T$ and $T_{\lambda}$ are both $\delta$ Jordan Lie supertriple systems.

For (3), we take $x_{1}, x_{2}, x_{3}, x_{4}, x_{5} \in T$ and calculate

$$
\begin{aligned}
& {\left[x_{1}, x_{2},\left[x_{3}, x_{4}, x_{5}\right]_{\lambda}\right]_{\lambda}=\left[x_{1}, x_{2},\left[x_{3}, x_{4}, x_{5}\right]\right.} \\
& \left.\quad+\lambda \psi\left(x_{3}, x_{4}, x_{5}\right)\right]_{\lambda}=\left[x_{1}, x_{2},\left[x_{3}, x_{4}, x_{5}\right]\right. \\
& \left.\quad+\lambda \psi\left(x_{3}, x_{4}, x_{5}\right)\right]+\lambda \psi\left(x_{1}, x_{2},\left[x_{3}, x_{4}, x_{5}\right]\right. \\
& \left.\quad+\lambda \psi\left(x_{3}, x_{4}, x_{5}\right)\right)=\left[x_{1}, x_{2},\left[x_{3}, x_{4}, x_{5}\right]\right] \\
& \quad+\lambda\left(\left[x_{1}, x_{2}, \psi\left(x_{3}, x_{4}, x_{5}\right)\right]\right. \\
& \left.\quad+\psi\left(x_{1}, x_{2},\left[x_{3}, x_{4}, x_{5}\right]\right)\right) \\
& \quad+\lambda^{2} \psi\left(x_{1}, x_{2}, \psi\left(x_{3}, x_{4}, x_{5}\right)\right)
\end{aligned}
$$

By similar calculation, we have

$$
\begin{aligned}
& {\left[\left[x_{1}, x_{2}, x_{3}\right]_{\lambda}, x_{4}, x_{5}\right]_{\lambda}=\left[\left[x_{1}, x_{2}, x_{3}\right], x_{4}, x_{5}\right]} \\
& \quad+\lambda\left(\left[\psi\left(x_{1}, x_{2}, x_{3}\right), x_{4}, x_{5}\right]\right. \\
& \left.\quad+\psi\left(\left[x_{1}, x_{2}, x_{3}\right], x_{4}, x_{5}\right)\right) \\
& \quad+\lambda^{2} \psi\left(\psi\left(x_{1}, x_{2}, x_{3}\right), x_{4}, x_{5}\right),
\end{aligned}
$$

$$
\begin{aligned}
& {\left[x_{3},\left[x_{1}, x_{2}, x_{4}\right]_{\lambda}, x_{5}\right]_{\lambda}=\left[x_{3},\left[x_{1}, x_{2}, x_{4}\right], x_{5}\right]} \\
& +\lambda\left(\left[x_{3}, \psi\left(x_{1}, x_{2}, x_{4}\right), x_{5}\right]\right. \\
& \left.\quad+\psi\left(x_{3},\left[x_{1}, x_{2}, x_{4}\right], x_{5}\right)\right) \\
& \quad+\lambda^{2} \psi\left(x_{3}, \psi\left(x_{1}, x_{2}, x_{4}\right), x_{5}\right), \\
& {\left[x_{3}, x_{4},\left[x_{1}, x_{2}, x_{5}\right]_{\lambda}\right]_{\lambda}=\left[x_{3}, x_{4},\left[x_{1}, x_{2}, x_{5}\right]\right]} \\
& \quad+\lambda\left(\left[x_{3}, x_{4}, \psi\left(x_{1}, x_{2}, x_{5}\right)\right]\right. \\
& \left.\quad+\psi\left(x_{3}, x_{4},\left[x_{1}, x_{2}, x_{5}\right]\right)\right) \\
& \quad+\lambda^{2} \psi\left(x_{3}, x_{4}, \psi\left(x_{1}, x_{2}, x_{5}\right)\right) .
\end{aligned}
$$

It follows that

$$
\begin{aligned}
& {\left[\left[x_{1}, x_{2}, x_{3}\right]_{\lambda}, x_{4}, x_{5}\right]_{\lambda}+(-1)^{\left|x_{3}\right|\left(\left|x_{1}\right|+\left|x_{2}\right|\right)}} \\
& \cdot\left[x_{3},\left[x_{1}, x_{2}, x_{4}\right]_{\lambda}, x_{5}\right]_{\lambda}+\delta(-1)^{\left(\left|x_{1}\right|+\left|x_{2}\right|\right)\left(\left|x_{3}\right|+\left|x_{4}\right|\right)} \\
& \cdot\left[x_{3}, x_{4},\left[x_{1}, x_{2}, x_{5}\right]_{\lambda}\right]_{\lambda}=\left[\left[x_{1}, x_{2}, x_{3}\right], x_{4}, x_{5}\right] \\
& +(-1)^{\left|x_{3}\right|\left(\left|x_{1}\right|+\left|x_{2}\right|\right)}\left[x_{3},\left[x_{1}, x_{2}, x_{4}\right], x_{5}\right] \\
& +\delta(-1)^{\left(\left|x_{1}\right|+\left|x_{2}\right|\right)\left(\left|x_{3}\right|+\left|x_{4}\right|\right)}\left[x_{3}, x_{4},\left[x_{1}, x_{2}, x_{5}\right]\right] \\
& +\lambda\left\{\left[\psi\left(x_{1}, x_{2}, x_{3}\right), x_{4}, x_{5}\right]\right. \\
& +\psi\left(\left[x_{1}, x_{2}, x_{3}\right], x_{4}, x_{5}\right)+(-1)^{\left|x_{3}\right|\left(\left|x_{1}\right|+\left|x_{2}\right|\right)} \\
& \cdot\left[x_{3}, \psi\left(x_{1}, x_{2}, x_{4}\right), x_{5}\right]+(-1)^{\left|x_{3}\right|\left(\left|x_{1}\right|+\left|x_{2}\right|\right)} \\
& \cdot \psi\left(x_{3},\left[x_{1}, x_{2}, x_{4}\right], x_{5}\right)+\delta(-1)^{\left(\left|x_{1}\right|+\left|x_{2}\right|\right)\left(\left|x_{3}\right|+\left|x_{4}\right|\right)} \\
& \cdot\left[x_{3}, x_{4}, \psi\left(x_{1}, x_{2}, x_{5}\right)\right]+\delta(-1)^{\left(\left|x_{1}\right|+\left|x_{2}\right|\right)\left(\left|x_{3}\right|+\left|x_{4} d\right|\right)} \\
& \left.\psi \psi\left(x_{3}, x_{4},\left[x_{1}, x_{2}, x_{5}\right]\right)\right\} \\
& +\lambda^{2}\left\{\psi\left(\psi\left(x_{1}, x_{2}, x_{3}\right), x_{4}, x_{5}\right)+(-1)^{\left|x_{3}\right|\left(\left|x_{1}\right|+\left|x_{2}\right|\right)}\right. \\
& \cdot \psi\left(x_{3}, \psi\left(x_{1}, x_{2}, x_{4}\right), x_{5}\right) \\
& +\delta(-1)^{\left(\left|x_{1}\right|+\left|x_{2}\right|\right)\left(\left|x_{3}\right|+\left|x_{4}\right|\right)} \\
& \left.\cdot \psi\left(x_{3}, x_{4}, \psi\left(x_{1}, x_{2}, x_{5}\right)\right)\right\} \text {. }
\end{aligned}
$$

Therefore, we have

$$
\begin{aligned}
& {\left[x_{1}, x_{2}, \psi\left(x_{3}, x_{4}, x_{5}\right)\right]+\psi\left(x_{1}, x_{2},\left[x_{3}, x_{4}, x_{5}\right]\right)} \\
& \quad=\left[\psi\left(x_{1}, x_{2}, x_{3}\right), x_{4}, x_{5}\right] \\
& \quad+\psi\left(\left[x_{1}, x_{2}, x_{3}\right], x_{4}, x_{5}\right)+(-1)^{\left|x_{3}\right|\left(\left|x_{1}\right|+\left|x_{2}\right|\right)} \\
& \quad \cdot\left[x_{3}, \psi\left(x_{1}, x_{2}, x_{4}\right), x_{5}\right]+(-1)^{\left|x_{3}\right|\left(\left|x_{1}\right|+\left|x_{2}\right|\right)} \\
& \quad \cdot \psi\left(x_{3},\left[x_{1}, x_{2}, x_{4}\right], x_{5}\right)+\delta(-1)^{\left(\left|x_{1}\right|+\left|x_{2}\right|\right)\left(\left|x_{3}\right|+\left|x_{4}\right|\right)}
\end{aligned}
$$




$$
\begin{aligned}
\cdot & {\left[x_{3}, x_{4}, \psi\left(x_{1}, x_{2}, x_{5}\right)\right]+\delta(-1)^{\left(\left|x_{1}\right|+\left|x_{2}\right|\right)\left(\left|x_{3}\right|+\left|x_{4}\right|\right)} } \\
\cdot & \psi\left(x_{3}, x_{4},\left[x_{1}, x_{2}, x_{5}\right]\right) \\
\psi & \left(x_{1}, x_{2}, \psi\left(x_{3}, x_{4}, x_{5}\right)\right)=\psi\left(\psi\left(x_{1}, x_{2}, x_{3}\right), x_{4}, x_{5}\right) \\
& +(-1)^{\left|x_{3}\right|\left(\left|x_{1}\right|+\left|x_{2}\right|\right)} \psi\left(x_{3}, \psi\left(x_{1}, x_{2}, x_{4}\right), x_{5}\right) \\
& +\delta(-1)^{\left(\left|x_{1}\right|+\left|x_{2}\right|\right)\left(\left|x_{3}\right|+\left|x_{4}\right|\right)} \psi\left(x_{3}, x_{4}, \psi\left(x_{1}, x_{2}, x_{5}\right)\right) .
\end{aligned}
$$

By (102), $\psi$ satisfies (3). So $\psi$ defines a $\delta$-Jordan Lie supertriple system structure on $T$.

Since $\theta\left(x_{1}, x_{2}\right)(x)=(-1)^{|x|\left(\left|x_{1}\right|+\left|x_{2}\right|\right)}\left[x, x_{1}, x_{2}\right]$ and $D\left(x_{1}, x_{2}\right)(x)=\delta\left[x_{1}, x_{2}, x\right]$, we can rewrite (97) as follows:

$$
\begin{aligned}
0= & -\delta D\left(x_{1}, x_{2}\right) \psi\left(x_{3}, x_{4}, x_{5}\right) \\
& -\psi\left(x_{1}, x_{2},\left[x_{3}, x_{4}, x_{5}\right]\right) \\
& +(-1)^{\left(\left|x_{1}\right|+\left|x_{2}\right|+\left|x_{3}\right|\right)\left(\left|x_{4}\right|+\left|x_{5}\right|\right)} \theta\left(x_{4}, x_{5}\right) \psi\left(x_{1}, x_{2}, x_{3}\right) \\
& +\psi\left(\left[x_{1}, x_{2}, x_{3}\right], x_{4}, x_{5}\right)-\delta(-1)^{\left|x_{3}\right|\left(\left|x_{1}\right|+\left|x_{2}\right|\right)} \\
& \cdot \theta\left(x_{3}, e\right) \psi\left(x_{1}, x_{2}, x_{4}\right)+(-1)^{\left|x_{3}\right|\left(\left|x_{1}\right|+\left|x_{2}\right|\right)} \\
& \cdot \psi\left(x_{3},\left[x_{1}, x_{2}, x_{4}\right], x_{5}\right)+\delta(-1)^{\left(\left|x_{1}\right|+\left|x_{2}\right|\right)\left(\left|x_{3}\right|+\left|x_{4}\right|\right)} \\
& \cdot D\left(x_{3}, x_{4}\right) \psi\left(x_{1}, x_{2}, x_{5}\right) \\
& +\delta(-1)^{\left(\left|x_{1}\right|+\left|x_{2}\right|\right)\left(\left|x_{3}\right|+\left|x_{4}\right|\right)} \psi\left(x_{3}, x_{4},\left[x_{1}, x_{2}, x_{5}\right]\right) \\
& =d^{3} \psi\left(x_{1}, x_{2}, x_{3}, x_{4}, x_{5}\right) .
\end{aligned}
$$

The last equality holds since $\psi$ is an even trilinear map. So $d^{3} \psi=0$, as required.

Conversely, if $\psi$ satisfies conditions (i) and (ii), it is easy to see that $T_{\lambda}$ is a $\delta$-Jordan Lie supertriple system from (96), (97), (101), and (102).

Definition 31. A deformation is said to be trivial if there exists a linear map $N: T \longrightarrow T$ such that, for all $\lambda, \varphi_{\lambda}=i d+\lambda N:$ $T_{\lambda} \longrightarrow T$ satisfies

$$
\varphi_{\lambda}\left[x_{1}, x_{2}, x_{3}\right]_{\lambda}=\left[\varphi_{\lambda} x_{1}, \varphi_{\lambda} x_{2}, \varphi_{\lambda} x_{3}\right],
$$

for any $x_{1}, x_{2}, x_{3} \in T$.

The left-hand side of (104) is equal to

$$
\begin{aligned}
& {\left[x_{1}, x_{2}, x_{3}\right]+\lambda\left\{\psi\left(x_{1}, x_{2}, x_{3}\right)+N\left[x_{1}, x_{2}, x_{3}\right]\right\}} \\
& \quad+\lambda^{2} N \psi\left(x_{1}, x_{2}, x_{3}\right) .
\end{aligned}
$$

The right-hand side of (104) is equal to

$$
\begin{aligned}
& {\left[x_{1}, x_{2}, x_{3}\right]+\lambda\left\{\left[N x_{1}, x_{2}, x_{3}\right]+\left[x_{1}, N x_{2}, x_{3}\right]\right.} \\
& \left.\quad+\left[x_{1}, x_{2}, N x_{3}\right]\right\}+\lambda^{2}\left\{\left[N x_{1}, N x_{2}, x_{3}\right]\right. \\
& \left.\left.\quad+\left[x_{1}, N x_{2}, N x_{3}\right]+\left[N x_{1}, x_{2}, N x_{3}\right)\right]\right\} \\
& \quad+\lambda^{3}\left[N x_{1}, N x_{2}, N x_{3}\right] .
\end{aligned}
$$

Therefore, by (104), we have

$$
0=\left[N x_{1}, N x_{2}, N x_{3}\right]
$$

$$
\begin{aligned}
N \psi\left(x_{1}, x_{2}, x_{3}\right)= & {\left[N x_{1}, N x_{2}, x_{3}\right]+\left[x_{1}, N x_{2}, N x_{3}\right] } \\
& \left.+\left[N x_{1}, x_{2}, N x_{3}\right)\right], \\
\psi\left(x_{1}, x_{2}, x_{3}\right)= & {\left[N x_{1}, x_{2}, x_{3}\right]+\left[x_{1}, N x_{2}, x_{3}\right] } \\
& +\left[x_{1}, x_{2}, N x_{3}\right]-N\left[x_{1}, x_{2}, x_{3}\right] \\
= & (-1)^{\left|x_{1}\right|\left(\left|x_{2}\right|+\left|x_{3}\right|\right)} \theta\left(x_{2}, x_{3}\right) N x_{1} \\
& -\delta(-1)^{\left|x_{2}\right|\left|x_{3}\right|} \theta\left(x_{1}, x_{3}\right) N x_{2} \\
& +\delta D\left(x_{1}, x_{2}\right) N x_{3} \\
& -N\left[x_{1}, x_{2}, x_{3}\right] \\
= & d^{1} N\left(x_{1}, x_{2}, x_{3}\right) .
\end{aligned}
$$

By (108) and (109), we can deduce that

$$
\begin{aligned}
N^{2}\left[x_{1}, x_{2}, x_{3}\right]= & N\left[N x_{1}, x_{2}, x_{3}\right]+N\left[x_{1}, N x_{2}, x_{3}\right] \\
& +N\left[x_{1}, x_{2}, N x_{3}\right] \\
& -\left[N x_{1}, N x_{2}, x_{3}\right] \\
& -\left[x_{1}, N x_{2}, N x_{3}\right] \\
& \left.-\left[N x_{1}, x_{2}, N x_{3}\right)\right] .
\end{aligned}
$$

Definition 32. A linear operator $N: T \longrightarrow T$ is called a Nijenhuis operator if and only if (107) and (110) hold.

Theorem 33. Let $N$ be a Nijenhuis operator for $T$. Then, a deformation of $T$ can be obtained by putting

$$
\begin{aligned}
\psi\left(x_{1}, x_{2}, x_{3}\right)= & (-1)^{\left|x_{1}\right|\left(\left|x_{2}\right|+\left|x_{3}\right|\right)} \theta\left(x_{2}, x_{3}\right) N x_{1} \\
& -\delta(-1)^{\left|x_{2}\right|\left|x_{3}\right|} \theta\left(x_{1}, x_{3}\right) N x_{2} \\
& +\delta D\left(x_{1}, x_{2}\right) N x_{3}-N\left[x_{1}, x_{2}, x_{3}\right] .
\end{aligned}
$$

Moreover, this deformation is trivial.

Proof. The proof is similar to one in the setting of $\delta$-Jordan Lie triple system in [14].

\section{Data Availability}

No data were used to support this study.

\section{Conflicts of Interest}

The authors declare that there are no conflicts of interest regarding the publication of this paper.

\section{Acknowledgments}

The paper is partially supported by the Anhui Provincial Natural Science Foundation (nos. 1908085MA03 and 
1808085MA14), the outstanding top-notch talent cultivation project of Anhui Province (no. gxfx2017123), the NSF of China (nos. 11761017 and 11801304), the Youth Project for Natural Science Foundation of Guizhou Provincial Department of Education (no. KY[2018]155), and the Project funded by China Postdoctoral Science Foundation (no. 2018M630768).

\section{References}

[1] N. Jacobson, "Lie and Jordan triple systems," American Journal of Mathematics, vol. 71, pp. 149-170, 1949.

[2] W. G. Lister, "A structure theory of Lie triple systems," Transactions of the American Mathematical Society, vol. 72, pp. 217-242, 1952.

[3] M. R. Bremner, R. Felipe, and J. Sánchez-Ortega, "Jordan triple disystems," Computers \& Mathematics with Applications, vol. 63, no. 6, pp. 1039-1055, 2012.

[4] B. Harris, "Cohomology of lie triple systems and lie algebras with involution," Transactions of the American Mathematical Society, vol. 98, no. 1, pp. 148-162, 1961.

[5] T. L. Hodge and B. J. Parshall, "On the representation theory of Lie triple systems," Transactions of the American Mathematical Society, vol. 354, no. 11, pp. 4359-4392, 2002.

[6] F. Kubo and Y. Taniguchi, "A controlling cohomology of the deformation theory of Lie triple systems," Journal of Algebra, vol. 278, no. 1, pp. 242-250, 2004.

[7] E. L. Stitzinger, "On derivation algebras of malcev algebras and lie triple systems," Proceedings of the American Mathematical Society, vol. 55, no. 1, pp. 9-13, 1976.

[8] K. Yamaguti, "On the cohomology space of Lie triple system," Kumamoto Journal of Science. Series A, vol. 5, pp. 44-52, 1960.

[9] Z. Zhang, L. Chen, W. Liu, and X. Bai, "The frattini subsystem of a lie triple system," Communications in Algebra, vol. 37, no. 10, pp. 3750-3759, 2009.

[10] T. Zhang, "Notes on cohomologies of Lie triple systems," Journal of Lie Theory, vol. 24, no. 4, pp. 909-929, 2014.

[11] Z. X. Zhang, Y. Q. Shi, and L. N. Zhao, "Invariant symmetric bilinear forms on lie triple systems," Communications in Algebra, vol. 30, no. 11, pp. 5563-5573, 2002.

[12] S. Okubo and N. Kamiya, "Jordan-lie super algebra and jordan-lie triple system," Journal of Algebra, vol. 198, no. 2, pp. 388-411, 1997.

[13] S. Okubo and N. Kamiya, "Quasi-classical Lie superalgebras and Lie supertriple systems," Communications in Algebra, vol. 30, no. 8, pp. 3825-3850, 2002.

[14] L. L. Ma and L. Y. Chen, “On $\delta$-Jordan Lie triple systems," Linear and Multilinear Algebra, vol. 65, no. 4, pp. 731-751, 2017.

[15] S. Okubo, "Parastatistics as Lie-supertriple systems," Journal of Mathematical Physics, vol. 35, no. 6, pp. 2785-2803, 1994.

[16] Y. Ma, L. Y. Chen, and D. Liu, "On generalized derivations of Lie supertriple systems," Acta Mathematica Sinica, vol. 56, no. 6, pp. 961-970, 2013.

[17] J. Peng, L. Chen, and B. Sun, "Centroids of lie supertriple systems," Advances in Mathematical Physics, vol. 2015, Article ID 949046, 9 pages, 2015.

[18] N. Kamiya, "A construction of anti-Lie triple systems from a class of triple systems," Shimane University. Faculty of Science. Memoirs, vol. 22, pp. 51-62, 1988. 


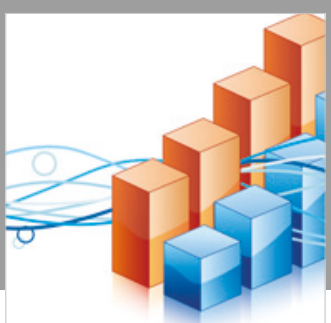

Advances in

Operations Research

\section{-n-m}
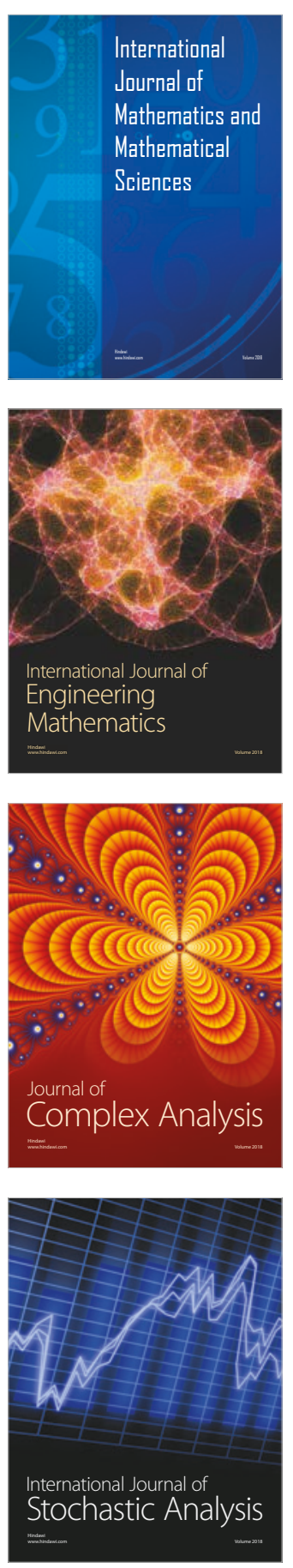
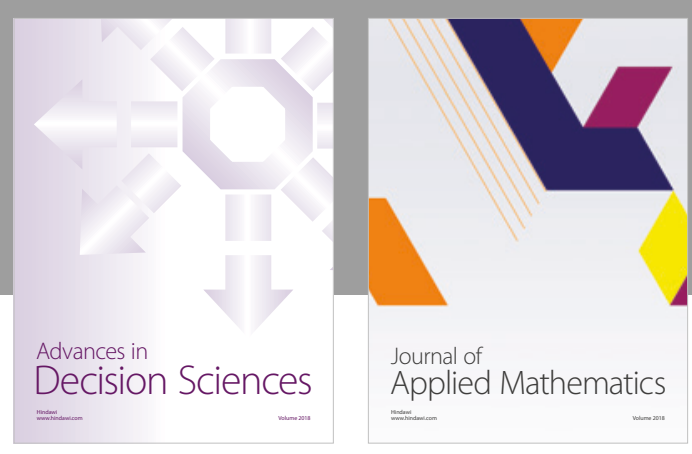

Journal of

Applied Mathematics
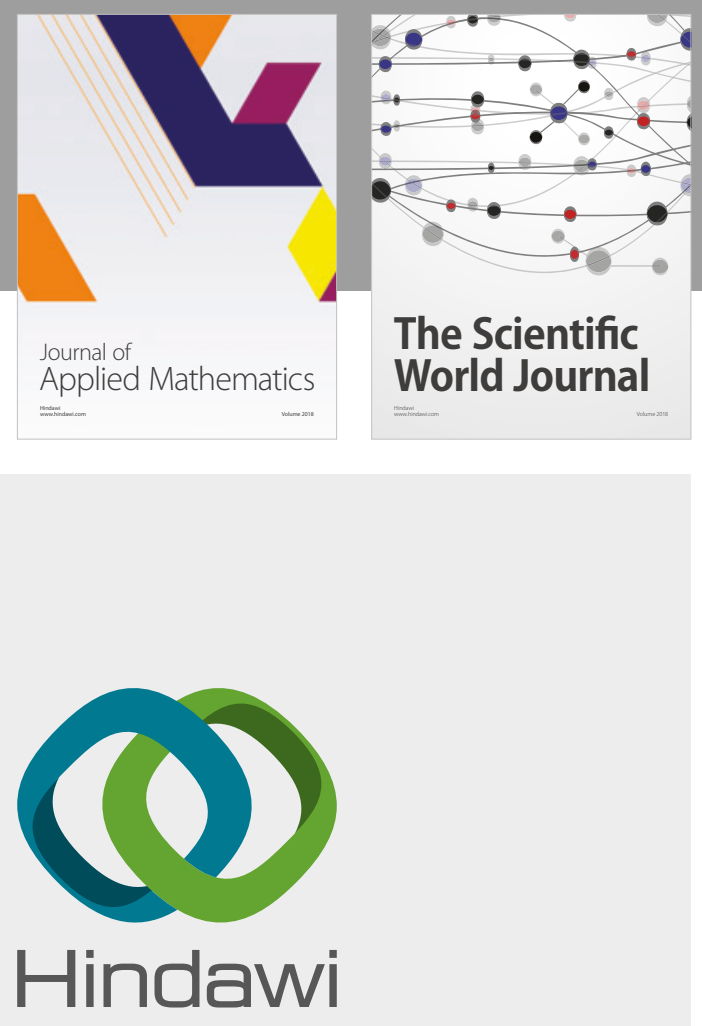

Submit your manuscripts at

www.hindawi.com

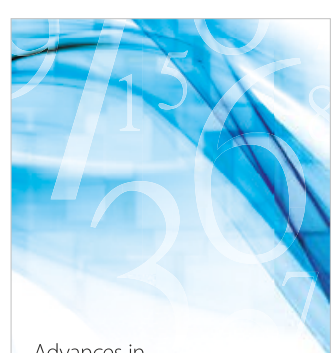

Advances in
Numerical Analysis
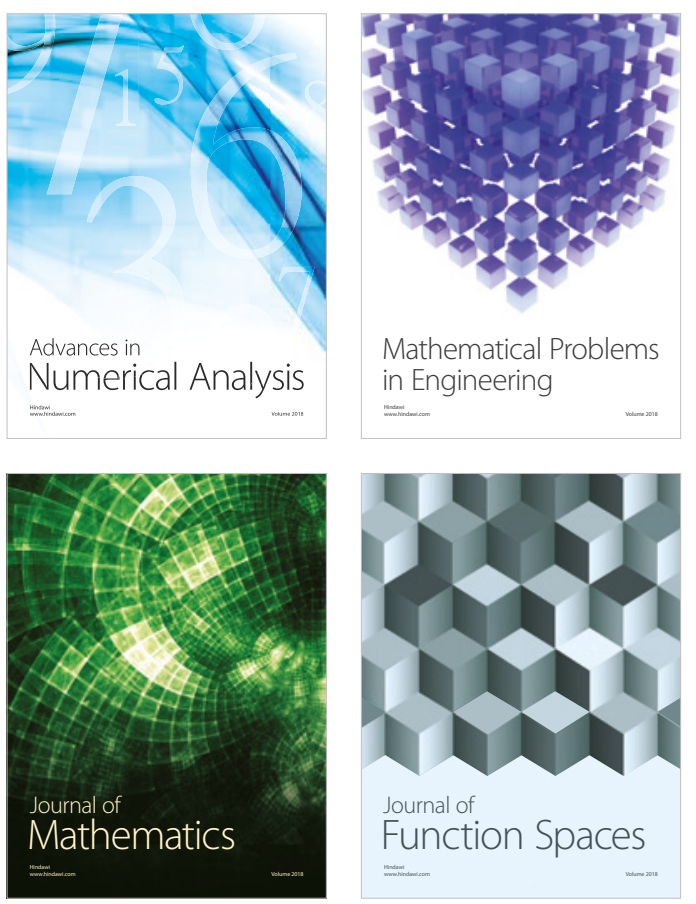

Mathematical Problems in Engineering

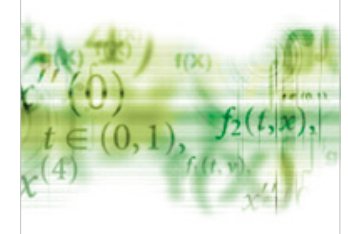

International Journal of

Differential Equations

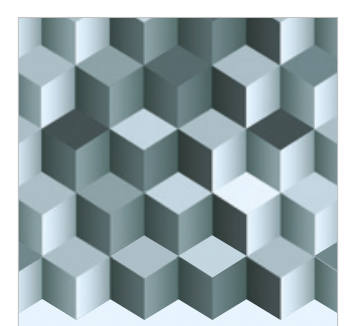

Journal of

Function Spaces

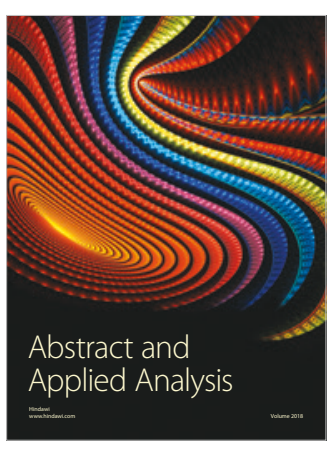

The Scientific

World Journal

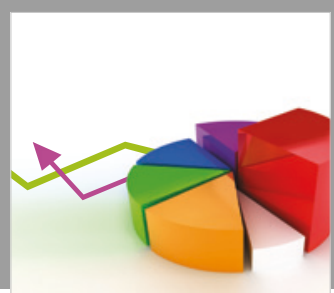

Journal of

Probability and Statistics
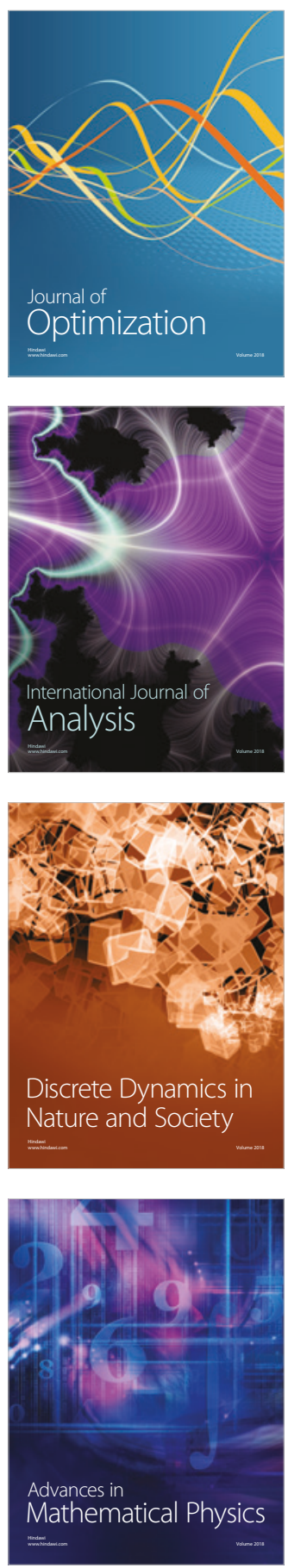